\title{
DECAY PROPERTY OF REGULARITY-LOSS TYPE FOR THE EULER-MAXWELL SYSTEM*
}

\author{
YOSHIHIRO $\mathrm{UEDA}^{\dagger}$ AND SHUICHI KAWASHIMA ${ }^{\ddagger}$
}

\begin{abstract}
We study the decay property for the Euler-Maxwell system in $\mathbb{R}^{3}$ and observe that it is of the regularity-loss type. We show that the solution decays at the rate $t^{-3 / 4}$ as $t \rightarrow \infty$, provided that the initial data are in $H^{s} \cap L^{1}$ with enough regularity $s \geq 6$. The proof is based on the time weighted energy method combined with the semigroup approach.
\end{abstract}

Key words. Euler-Maxwell system, decay estimate, regularity-loss, energy method.

AMS subject classifications. 35B35, 35L40, 35B40, 82D10.

1. Introduction. We consider the Euler-Maxwell system in $\mathbb{R}^{3}$ which appears in plasma physics (see, for example, $[2,3,19])$ :

$$
\left\{\begin{array}{l}
n_{t}+\operatorname{div}(n u)=0 \\
(n u)_{t}+\operatorname{div}(n u \otimes u)+\nabla p(n)=-n(E+u \times B)-n u \\
E_{t}-\operatorname{rot} B=n u \\
B_{t}+\operatorname{rot} E=0
\end{array}\right.
$$

$$
\operatorname{div} E=n_{\infty}-n
$$

$$
\operatorname{div} B=0
$$

Here $n>0$ and $u \in \mathbb{R}^{3}$ are the density and the velocity of the electron, respectively, while $E \in \mathbb{R}^{3}$ is the electric field and $B \in \mathbb{R}^{3}$ is the magnetic induction; these are unknown functions of $t>0$ and $x \in \mathbb{R}^{3}$. On the other hand, the pressure $p(n)$ is a given smooth function of $n$ satisfying $p^{\prime}(n)>0$ for $n>0$, and $n_{\infty}>0$ is a constant denoting the ion density.

We note that the system (1.1) is regarded as a symmetric hyperbolic system. Also, the system (1.1) with (1.2) and (1.3) admits a constant equilibrium state given by

$$
(n, u, E, B)=\left(n_{\infty}, 0,0, B_{\infty}\right)
$$

where $B_{\infty} \in \mathbb{R}^{3}$ is an arbitrarily fixed constant vector. In this paper we study (1.1), (1.2), (1.3) with the initial condition

$$
(n, u, E, B)(0, x)=\left(n_{0}, u_{0}, E_{0}, B_{0}\right)(x) .
$$

We see that (1.2) and (1.3), which are regarded as the constraints, hold true for any $t>0$ if they hold initially. In fact, if the initial data verify

$$
\operatorname{div} E_{0}=n_{\infty}-n_{0}, \quad \operatorname{div} B_{0}=0
$$

\footnotetext{
*Received January 5, 2011; accepted for publication August 3, 2011.

${ }^{\dagger}$ Faculty of Maritime Sciences, Kobe University, Kobe 658-0022, Japan (ueda@maritime.kobeu.ac.jp).

${ }^{\ddagger}$ Faculty of Mathematics, Kyushu University, Fukuoka 819-0395, Japan (kawashim@math.kyushuu.ac.jp).
} 
then the solutions to the problem (1.1), (1.5) always satisfy (1.2) and (1.3) for all $t>0$.

The initial value problem (1.1), (1.5) was first studied by Peng, Wang and Gu [19]. They considered the problem with periodic boundary conditions and proved the global existence of solutions $(n, u, E, B)(t, x)$ in a small $H^{4}$ neighborhood of the constant equilibrium state $\left(n_{\infty}, 0,0, B_{\infty}\right)$. Also, they verified the $L^{\infty}$ convergence of $(n, u)(t, x)$ toward $\left(n_{\infty}, 0\right)$ as $t \rightarrow \infty$. On the other hand, the pure initial value problem (1.1), (1.5) was discussed in our previous paper [23]. It was proved in [23] that a unique global solution $(n, u, E, B)(t, x)$ exists in a small $H^{s}(s \geq 3)$ neighborhood of the equilibrium state $\left(n_{\infty}, 0,0, B_{\infty}\right)$ and converges to $\left(n_{\infty}, 0,0, B_{\infty}\right)$ in $L^{\infty}$ norm as $t \rightarrow \infty$. This is a refinement of the result in [19].

The main purpose of this paper is to show the quantitative decay estimates of solutions toward the equilibrium state $\left(n_{\infty}, 0,0, B_{\infty}\right)$. First, under the smallness condition on $I_{0}=\left\|\left(n_{0}-n_{\infty}, u_{0}, E_{0}, B_{0}-B_{\infty}\right)\right\|_{H^{s}}$, where $s \geq 6$, we show that

$$
\left\|\partial_{x}^{k}\left(n-n_{\infty}, u, E, B-B_{\infty}\right)(t)\right\|_{H^{s-2 k}} \leq C I_{0}(1+t)^{-k / 2}
$$

for each $k$ with $0 \leq k \leq[s / 2]$ (see Theorem 2.2). Moreover, when $I_{1}=\|\left(n_{0}-\right.$ $\left.n_{\infty}, u_{0}, E_{0}, B_{0}-B_{\infty}\right) \|_{H^{s} \cap L^{1}}$ with $s \geq 6$ is small, we have the faster decay estimate

$$
\left\|\partial_{x}^{k}\left(n-n_{\infty}, u, E, B-B_{\infty}\right)(t)\right\|_{H^{s-2 k-3}} \leq C I_{1}(1+t)^{-3 / 4-k / 2}
$$

for each $k$ with $0 \leq k \leq[(s-1) / 2]-1$ (see Theorem 2.3). The decay estimate (1.7) is obtained for initial perturbation in $H^{s}$ and is based on the time weighted energy method. On the other hand, the faster decay estimate (1.8) is shown for initial perturbation in $H^{s} \cap L^{1}$ and is obtained by a combination of the time weighted energy method and the semigroup argument which makes use of the decay estimate for the linearized problem (see Corollary 5.2).

As observed in [23], the dissipative structure of our system $(1.1),(1.2),(1.3)$ is weaker than the standard one characterized in $[20,22,10]$ and is of the regularityloss type. In fact, we have the regularity-loss not only in the dissipation part of the energy estimate but also in the decay estimate for the linearized system. This weaker dissipation causes additional difficulties in establishing a global existence result and especially in obtaining the time asymptotic decay of solutions. A similar dissipative structure of the regularity-loss type was also found for various systems of partial differential equations. See [16, 8, 9] for the dissipative Timoshenko system, [7, 13] for hyperbolic-elliptic systems related to the radiation hydrodynamics, and $[4,21,14,15]$ for a dissipative plate equation.

There are many works on the global existence and asymptotic stability of solutions to the initial value problem for symmetric hyperbolic systems with dissipation. We refer the reader to $[6,24,1,12]$. All these works were, however, based on the standard dissipative structure formulated in $[20,22,10]$ and hence are different from the present paper. Also, there are some interesting results on global existence and asymptotic limit for small parameters for the Euler-Maxwell system (1.1), (1.2), (1.3). We refer the reader to $[3,17,18]$ and references therein.

The contents of this paper are as follows. In Section 2, we summarize some basic properties of our system and give the statement of our main theorems. In Section 3 , we review the energy method employed in [23]. Then, in Section 4, we develop the time weighted energy method and give the proof of Theorem 2.2 on the global existence and asymptotic decay of solutions for initial perturbation in $H^{s}$. In Section 5 , we study the decay property of the linearized system and prove the decay estimate 
for the semigroup associated with the linearized problem. This decay estimate is based on the pointwise estimate in the Fourier space. Finally, in Section 6, by applying the semigroup argument, we prove Theorem 2.3 on the faster decay estimate (1.8) for initial perturbation in $H^{s} \cap L^{1}$.

Added in proof. Through the private communication with Professor R. Duan, we have learned that he obtained the decay estimate (1.8) under the regularity assumption $s \geq 13$ on the initial perturbation. See [5] for the details. We remark that our time weighted energy method enables us to prove the same decay estimate under the less-regularity assumption $s \geq 6$.

Notations. We use the standard notations $\nabla=\left(\partial_{x_{1}}, \partial_{x_{2}}, \partial_{x_{3}}\right)$ and $\Delta=$ $\sum_{j=1}^{3} \partial_{x_{j}}^{2}$ for the gradient and Laplacian with respect to $x=\left(x_{1}, x_{2}, x_{3}\right)$, respectively. Also, we use the divergence and rotation: $\operatorname{div} u=\sum_{j=1}^{3} \partial_{x_{j}} u_{j}$ and $\operatorname{rot} u=$ $\left(\partial_{x_{2}} u_{3}-\partial_{x_{3}} u_{2}, \partial_{x_{3}} u_{1}-\partial_{x_{1}} u_{3}, \partial_{x_{1}} u_{2}-\partial_{x_{1}} u_{2}\right)$ for a vector function $u=\left(u_{1}, u_{2}, u_{3}\right)$. For a nonnegative integer $k$, we denote by $\partial_{x}^{k}$ the totality of all the $k$-th order derivatives with respect to $x=\left(x_{1}, x_{2}, x_{3}\right)$.

Let $1 \leq p \leq \infty$. Then $L^{p}=L^{p}\left(\mathbb{R}^{3}\right)$ denotes the usual Lebesgue space over $\mathbb{R}^{3}$ with the norm $\|\cdot\|_{L^{p}}$. For a nonnegative integer $s, W^{s, p}=W^{s, p}\left(\mathbb{R}^{3}\right)$ denotes the $s$-th order Sobolev space over $\mathbb{R}^{3}$ in the $L^{p}$ sense, equipped with the norm $\|\cdot\|_{W^{s, p}}$. When $p=2$, we use the abbreviation $H^{s}=W^{s, 2}$. We note that $L^{p}=W^{0, p}$ and $L^{2}=H^{0}$. Let $I$ be an interval in $[0, \infty)$ and $X$ be a Banach space over $\mathbb{R}^{3}$. Then, for a nonnegative integer $k, C^{k}(I ; X)$ denotes the space of $k$-times continuously differential functions on $I$ with values in $X$.

Finally, in this paper, we use $C$ or $c$ to denote various positive constants without confusion.

2. Main results. Before stating our main results, we briefly summarize the basic properties of the Euler-Maxwell system (1.1). First, we observe that the system (1.1) is written as

$$
\left\{\begin{array}{l}
n_{t}+u \cdot \nabla n+n \operatorname{div} u=0, \\
u_{t}+(n \cdot \nabla) u+a(n) \nabla n+E+u \times B+u=0, \\
E_{t}-\operatorname{rot} B-n u=0, \\
B_{t}+\operatorname{rot} E=0,
\end{array}\right.
$$

where $a(n)=p^{\prime}(n) / n$. We put $w=(n, u, E, B)^{T} ; w$ is a column vector in $\mathbb{R}^{10}$ and the superscript " $T$ " denotes the transposed. Then the system (2.1) is rewritten in the vector form as

$$
A^{0}(w) w_{t}+\sum_{j=1}^{3} A^{j}(w) w_{x_{j}}+L(w) w=0
$$


where the coefficient matrices are given explicitly as

$$
A^{0}(w)=\left(\begin{array}{cccc}
a(n) & & & \\
& n I & & \\
& & I & \\
& & & I
\end{array}\right), \quad L(w)=\left(\begin{array}{cccc}
0 & & & \\
& n\left(I-\Omega_{B}\right) & n I & \\
-n I & O & \\
& & & O
\end{array}\right),
$$

$$
\sum_{j=1}^{3} A^{j}(w) \xi_{j}=\left(\begin{array}{cccc}
a(n)(u \cdot \xi) & p^{\prime}(n) \xi & & \\
p^{\prime}(n) \xi^{T} & n(u \cdot \xi) I & & \\
& & O & -\Omega_{\xi} \\
& & \Omega_{\xi} & O
\end{array}\right)
$$

Here $I$ and $O$ denotes the $3 \times 3$ identity matrix and the zero matrix, respectively, $\xi=\left(\xi_{1}, \xi_{2}, \xi_{3}\right) \in \mathbb{R}^{3}$, and $\Omega_{\xi}$ is the skew-symmetric matrix defined by

$$
\Omega_{\xi}=\left(\begin{array}{ccc}
0 & -\xi_{3} & \xi_{2} \\
\xi_{3} & 0 & -\xi_{1} \\
-\xi_{2} & \xi_{1} & 0
\end{array}\right)
$$

for $\xi=\left(\xi_{1}, \xi_{2}, \xi_{3}\right) \in \mathbb{R}^{3}$, so that we have $\Omega_{\xi} E^{T}=(\xi \times E)^{T}$ (as a column vector in $\left.\mathbb{R}^{3}\right)$ for $E=\left(E_{1}, E_{2}, E_{3}\right) \in \mathbb{R}^{3}$. It should be noted that (2.2) is a symmetric hyperbolic system because $A^{0}(w)$ is real symmetric and positive definite and $A^{j}(w)$, $j=1,2,3$, are real symmetric. Also, the matrix $L(w)$ is nonnegative definite, so that it is regarded as a dissipation matrix. However, the matrix $L(w)$ or $L\left(w_{\infty}\right)$ has skew-symmetric part and is not real symmetric, where $w_{\infty}=\left(n_{\infty}, 0,0, B_{\infty}\right)^{T}$ is the constant state in (1.4); notice that we have $L(w) w_{\infty}=0$ for each $w$ so that the constant state $w_{\infty}$ lies in the kernel of $L(w)$. Consequently, our system $(2.2)$ is not included in a class of systems considered in $[1,6,10,11,12,20,22,24]$.

Next, we discuss the dissipative structure of the system (1.1) together with (1.3); the equation (1.2) is not considered here. The linearization of (1.1), (1.3) around the constant state $w_{\infty}$ is given by

$$
\begin{gathered}
A^{0} z_{t}+\sum_{j=1}^{3} A^{j} z_{x_{j}}+L z=0, \\
\sum_{j=1}^{3} R^{j} z_{x_{j}}=0
\end{gathered}
$$

where $z=w-w_{\infty}$. Here we simply wrote as $A^{0}=A^{0}\left(w_{\infty}\right), A^{j}=A^{j}\left(w_{\infty}\right)$ and $L=L\left(w_{\infty}\right)$, while $R^{j}$ are $1 \times 10$ matrices given by $R(\xi)=\sum_{j=1}^{3} R^{j} \xi_{j}=(0,0,0, \xi)$ for $\xi=\left(\xi_{1}, \xi_{2}, \xi_{3}\right) \in \mathbb{R}^{3}$. We apply the Fourier transform to (2.4) and (2.5) and obtain

$$
\begin{gathered}
A^{0} \hat{z}_{t}+i|\xi| A(\omega) \hat{z}+L \hat{z}=0, \\
i|\xi| R(\omega) \hat{z}=0,
\end{gathered}
$$

where $A(\omega)=\sum_{j=1}^{3} A^{j} \omega_{j}$ and $\omega=\xi /|\xi| \in S^{2}$. The stability condition for the system (2.4) with (2.5) is then formulated as follows (see [20, 10, 22]). 
Stability condition [SC]: Let $\omega \in S^{2}$ and $\mu \in \mathbb{R}$. Suppose that $\varphi \in \mathbb{C}^{10}$ satisfies $L \varphi=0, \mu A^{0} \varphi+A(\omega) \varphi=0$, and $R(\omega) \varphi=0$. Then we have $\varphi=0$.

Here we impose the restriction $R(\omega) \varphi=0$ to treat the equation (2.5) and this is motivated by the work [22]. We can check that our system verifies the stability condition formulated above; for the details, we refer the reader to [23]. However, the dissipation matrix $L$ is not real symmetric and therefore we can not apply the general theory on the dissipative structure developed in $[20,22,10,6,24,11,1,12]$ to our system. This situation is quite similar to that for the dissipative Timoshenko system. We refer the reader to $[8,9]$.

In what follows we use the vector notations:

$$
w=(n, u, E, B)^{T}, \quad w_{\infty}=\left(n_{\infty}, 0,0, B_{\infty}\right)^{T}, \quad w_{0}=\left(n_{0}, u_{0}, E_{0}, B_{0}\right)^{T},
$$

were the superscript " $T$ " denotes the transposed. These functions are regarded as column vectors in $\mathbb{R}^{10}$. Then we state our main theorems concerning the decay rate of solutions to the initial value problem (1.1), (1.5). First, we summarize the main result in [23] on the time global existence and asymptotic decay of solutions. We use the following energy norm $N_{0}(t)$ and the corresponding dissipation norm $D_{0}(t)$ :

$$
\begin{aligned}
N_{0}(t) & :=\sup _{0 \leq \tau \leq t}\left\|\left(w-w_{\infty}\right)(\tau)\right\|_{H^{s}}, \\
D_{0}(t)^{2} & :=\int_{0}^{t}\left(\left\|\left(n-n_{\infty}, u\right)(\tau)\right\|_{H^{s}}^{2}+\|E(\tau)\|_{H^{s-1}}^{2}+\left\|\partial_{x} B(\tau)\right\|_{H^{s-2}}^{2}\right) d \tau .
\end{aligned}
$$

Theorem 2.1 (Global existence ([23])). Let $s \geq 3$ and suppose that the initial data satisfy $w_{0}-w_{\infty} \in H^{s}$ and (1.6). Put $I_{0}=\left\|w_{0}-w_{\infty}\right\|_{H^{s}}$. Then there exists a positive constant $\varepsilon_{0}$ such that if $I_{0} \leq \varepsilon_{0}$, then the initial value problem (1.1), (1.5) has a unique global solution $w(t, x)$ with $w-w_{\infty} \in C\left([0, \infty) ; H^{s}\right) \cap C^{1}\left([0, \infty) ; H^{s-1}\right)$. The solution satisfies the uniform energy estimate

$$
N_{0}(t)^{2}+D_{0}(t)^{2} \leq C I_{0}^{2}
$$

for $t \geq 0$. Moreover, the solution $w(t, x)$ converges to the constant state $w_{\infty}$ uniformly in $x \in \mathbb{R}^{3}$ as $t \rightarrow \infty$. More precisely, we have

$$
\begin{aligned}
& \left\|\left(n-n_{\infty}, u, E\right)(t)\right\|_{W^{s-2, \infty}} \rightarrow 0, \\
& \left\|\left(B-B_{\infty}\right)(t)\right\|_{W^{s-4, \infty}} \rightarrow 0
\end{aligned}
$$

as $t \rightarrow \infty$, where the asymptotic convergence for $B$ holds true only by assuming the additional regularity $s \geq 4$.

REMARK. We note that the uniform energy estimate (2.6) is of the regularity-loss type because we have 1-regularity loss for $(E, B)$ in the dissipation part $D_{0}(t)$ of $(2.6)$.

For the solution constructed in Theorem 2.1, we can derive the quantitive decay estimate. To state the result precisely, we introduce the following time weighted 
energy norm $N(t)$ and the corresponding dissipation norm $D(t)$ :

$$
\begin{aligned}
N(t)^{2} & :=\sum_{k=0}^{[s / 2]} \sup _{0 \leq \tau \leq t}(1+\tau)^{k}\left\|\partial_{x}^{k}\left(w-w_{\infty}\right)(\tau)\right\|_{H^{s-2 k}}^{2} \\
D(t)^{2} & :=\sum_{k=0}^{[s / 2]} \int_{0}^{t}(1+\tau)^{k}\left\|\partial_{x}^{k} u(\tau)\right\|_{H^{s-2 k}}^{2} d \tau+\sum_{k=0}^{[s / 2]-1} \int_{0}^{t}(1+\tau)^{k} \tilde{D}_{k}(\tau)^{2} d \tau,
\end{aligned}
$$

where

$$
\tilde{D}_{k}(t)^{2}:=\left\|\partial_{x}^{k}\left(n-n_{\infty}, u\right)(t)\right\|_{H^{s-2 k}}^{2}+\left\|\partial_{x}^{k} E(t)\right\|_{H^{s-2 k-1}}^{2}+\left\|\partial_{x}^{k+1} B(t)\right\|_{H^{s-2 k-2}}^{2} .
$$

Also, we use the following support norm $N_{\perp}(t)$ :

$$
N_{\perp}(t):=\sum_{k=0}^{[s / 2]-1} \sup _{0 \leq \tau \leq t}(1+t)^{1 / 2+k / 2}\left\|\partial_{x}^{k}\left(n-n_{\infty}, u, E\right)(t)\right\|_{H^{s-2 k-2}} .
$$

Then our first main result in this paper is stated as follows.

ThEOREM 2.2 (Decay estimate). Let $s \geq 6$ and suppose that the initial data satisfy $w_{0}-w_{\infty} \in H^{s}$ and (1.6). Then there exists a positive constant $\varepsilon_{1}$ such that if $I_{0} \leq \varepsilon_{1}$, then the global solution constructed in Theorem 2.1 satisfies the time weighted energy estimate

$$
N(t)^{2}+D(t)^{2}+N_{\perp}(t)^{2} \leq C I_{0}^{2}
$$

for $t \geq 0$. In particular, we have the following quantitative decay estimates:

$$
\begin{gathered}
\left\|\partial_{x}^{k}\left(w-w_{\infty}\right)(t)\right\|_{H^{s-2 k}} \leq C I_{0}(1+t)^{-k / 2}, \\
\left\|\partial_{x}^{k}\left(n-n_{\infty}, u, E\right)(t)\right\|_{H^{s-2 k-2}} \leq C I_{0}(1+t)^{-1 / 2-k / 2}, \\
\left\|\partial_{x}^{k}\left(n-n_{\infty}\right)(t)\right\|_{H^{s-2 k-4}} \leq C I_{0}(1+t)^{-1-k / 2},
\end{gathered}
$$

where $0 \leq k \leq[s / 2]$ in (2.11), $0 \leq k \leq[s / 2]-1$ in (2.12), and $0 \leq k \leq[s / 2]-2$ in $(2.13)$.

Moreover, under the additional condition $w_{0}-w_{\infty} \in L^{1}$, we can show the faster decay estimate. We introduce the following time weighted norm:

$$
M(t):=\sum_{k=0}^{[(s-1) / 2]-1} \sup _{0 \leq \tau \leq t}(1+\tau)^{3 / 4+k / 2}\left\|\partial_{x}^{k}\left(w-w_{\infty}\right)(\tau)\right\|_{H^{s-2 k-3}},
$$

that is corresponding to the optimal decay for lower order derivatives of the solution. Our second main result in this paper is then stated as follows.

THEOREM 2.3 (Faster decay estimate). Let $s \geq 6$ and suppose that the initial data satisfy $w_{0}-w_{\infty} \in H^{s} \cap L^{1}$ and (1.6). Put $I_{1}=\left\|w_{0}-w_{\infty}\right\|_{H^{s}}+\left\|w_{0}-w_{\infty}\right\|_{L^{1}}$. Then there exists a positive constant $\varepsilon_{2}$ such that if $I_{1} \leq \varepsilon_{2}$, then the global solution constructed in Theorem 2.1 satisfies the estimate

$$
M(t) \leq C I_{1}
$$


for $t \geq 0$. In particular, we have

$$
\begin{gathered}
\left\|\partial_{x}^{k}\left(w-w_{\infty}\right)(t)\right\|_{H^{s-2 k-3}} \leq C I_{1}(1+t)^{-3 / 4-k / 2}, \\
\left\|\partial_{x}^{k}\left(n-n_{\infty}, u, E\right)(t)\right\|_{H^{s-2 k-5}} \leq C I_{1}(1+t)^{-5 / 4-k / 2}, \\
\left\|\partial_{x}^{k}\left(n-n_{\infty}\right)(t)\right\|_{H^{s-2 k-7}} \leq C I_{1}(1+t)^{-7 / 4-k / 2},
\end{gathered}
$$

where $0 \leq k \leq[(s-1) / 2]-1$ in $(2.16), 0 \leq k \leq[(s-1) / 2]-2$ in (2.17), and $s \geq 7$ and $0 \leq k \leq[(s-1) / 2]-3$ in $(2.18)$.

3. Energy method. In this section, we review the energy method employed in [23] for the Euler-Maxwell system (1.1), (1.2), (1.3). This will be useful in introducing our time weighted energy method in the next section.

In the following, we consider solutions satisfying

$$
\left\|\left(w-w_{\infty}\right)(t)\right\|_{L^{\infty}} \leq \bar{\varepsilon}
$$

where $\bar{\varepsilon}$ is a suitably small positive constant. Let $0 \leq \ell \leq s$. We apply $\partial_{x}^{\ell}$ to (2.1). The result is written as

$$
\left\{\begin{array}{l}
\partial_{x}^{\ell} n_{t}+u \cdot \nabla \partial_{x}^{\ell} n+n \operatorname{div} \partial_{x}^{\ell} u=f_{1}^{(\ell)} \\
\partial_{x}^{\ell} u_{t}+(n \cdot \nabla) \partial_{x}^{\ell} u+a(n) \nabla \partial_{x}^{\ell} n+\partial_{x}^{\ell} E+\partial_{x}^{\ell} u \times B+\partial_{x}^{\ell} u=f_{2}^{(\ell)}+\tilde{f}_{2}^{(\ell)} \\
\partial_{x}^{\ell} E_{t}-\operatorname{rot} \partial_{x}^{\ell} B-n \partial_{x}^{\ell} u=\tilde{f}_{3}^{(\ell)} \\
\partial_{x}^{\ell} B_{t}+\operatorname{rot} \partial_{x}^{\ell} E=0
\end{array}\right.
$$

where we put

$$
\begin{array}{ll}
f_{1}^{(\ell)}=-\left[\partial_{x}^{\ell}, u \cdot \nabla\right] n-\left[\partial_{x}^{\ell}, n \operatorname{div}\right] u, & f_{2}^{(\ell)}=-\left[\partial_{x}^{\ell},(u \cdot \nabla)\right] u-\left[\partial_{x}^{\ell}, a(n) \nabla\right] n, \\
\tilde{f}_{2}^{(\ell)}=-\left\{\partial_{x}^{\ell}(u \times B)-\partial_{x}^{\ell} u \times B\right\}, & \tilde{f}_{3}^{(\ell)}=\partial_{x}^{\ell}(n u)-n \partial_{x}^{\ell} u,
\end{array}
$$

and $[$,$] denotes the commutator defined by [A, B]=A B-B A$. The system (3.2) is written as

$$
A^{0}(w) \partial_{x}^{\ell} w_{t}+\sum_{j=1}^{3} A^{j}(w) \partial_{x}^{\ell} w_{x_{j}}+L(w) \partial_{x}^{\ell} w=f_{A}^{(\ell)}+f_{L}^{(\ell)},
$$

where $f_{A}^{(\ell)}=\left(a(n) f_{1}^{(\ell)}, n f_{2}^{(\ell)}, 0,0\right)^{T}$ and $f_{L}^{(\ell)}=\left(0, n \tilde{f}_{2}^{(\ell)}, \tilde{f}_{3}^{(\ell)}, 0\right)^{T}$. Notice that $f_{A}^{(\ell)}=$ $f_{L}^{(\ell)}=0$ for $\ell=0$. The system (3.3) is regarded as a symmetric hyperbolic system for $\partial_{x}^{\ell} w$. We take the inner product of (3.3) with $\partial_{x}^{\ell}\left(w-w_{\infty}\right)$ and integrate the resultant equation with respect to $x \in \mathbb{R}^{3}$. This yields the standard energy inequality

$$
\frac{d}{d t} H_{0}^{(\ell)}+c\left\|\partial_{x}^{\ell} u\right\|_{L^{2}}^{2} \leq R_{A}^{(\ell)}+R_{L}^{(\ell)}
$$

where $H_{0}^{(\ell)}=\int_{\mathbb{R}^{3}}\left\langle A^{0}(w) \partial_{x}^{\ell}\left(w-w_{\infty}\right), \partial_{x}^{\ell}\left(w-w_{\infty}\right)\right\rangle d x$. We see that $R_{L}^{(\ell)}=0$ if $\ell=0$, which is due to the fact that $f_{L}^{(\ell)}=0$ for $\ell=0$. The technical computations as in [23] show that

$$
\begin{aligned}
c\left\|\partial_{x}^{\ell}\left(w-w_{\infty}\right)\right\|_{L^{2}}^{2} \leq H_{0}^{(\ell)} \leq C\left\|\partial_{x}^{\ell}\left(w-w_{\infty}\right)\right\|_{L^{2}}^{2}, \\
R_{A}^{(\ell)} \leq C\left\|\partial_{x}(n, u)\right\|_{L^{\infty}}\left\|\partial_{x}^{\ell}\left(n-n_{\infty}, u\right)\right\|_{L^{2}}^{2}, \\
R_{L}^{(\ell)} \leq C\left\|B-B_{\infty}\right\|_{L^{\infty}}\left\|\partial_{x}^{\ell} u\right\|_{L^{2}}^{2} \\
\quad+C\left\|\left(n-n_{\infty}, u\right)\right\|_{L^{\infty}}\left\|\partial_{x}^{\ell}\left(n-n_{\infty}, u\right)\right\|_{L^{2}}\left\|\partial_{x}^{\ell}\left(E, B-B_{\infty}\right)\right\|_{L^{2}}
\end{aligned}
$$


for $0 \leq \ell \leq s$.

Next we rewrite the system (2.1) as

$$
\left\{\begin{array}{l}
n_{t}+n_{\infty} \operatorname{div} u=g_{1}, \\
u_{t}+a_{\infty} \nabla n+E+u \times B_{\infty}+u=g_{2}, \\
E_{t}-\operatorname{rot} B-n_{\infty} u=g_{3}, \\
B_{t}+\operatorname{rot} E=0,
\end{array}\right.
$$

where $a_{\infty}=a\left(n_{\infty}\right)$ with $a(n)=p^{\prime}(n) / n$ and

$$
\begin{aligned}
& g_{1}=-u \cdot \nabla n-\left(n-n_{\infty}\right) \operatorname{div} u, \\
& g_{2}=-(u \cdot \nabla) u-\left(a(n)-a_{\infty}\right) \nabla n-u \times\left(B-B_{\infty}\right), \\
& g_{3}=\left(n-n_{\infty}\right) u .
\end{aligned}
$$

We regard (3.6) as a linear system for $w$ with constant coefficient. Now, letting $0 \leq \ell \leq s-1$, we apply $\partial_{x}^{\ell}$ to (3.6) to get the system for $\partial_{x}^{\ell} w$; we denote this system for $\partial_{x}^{\ell} w$ by $\partial_{x}^{\ell}(3.6)$. We create the dissipation terms by using the system $\partial_{x}^{\ell}(3.6)$. First, we multiply the first equation of $\partial_{x}^{\ell}(3.6)$ by $-a_{\infty} \operatorname{div} \partial_{x}^{\ell} u$, and take the inner product of the second and the third equations with $a_{\infty} \nabla \partial_{x}^{\ell} n+\partial_{x}^{\ell} E$ and $\partial_{x}^{\ell} u$, respectively. Then we add these three equations and integrate over $x \in \mathbb{R}^{3}$. The technical computations in [23], which make use of (1.2), give

$$
\frac{d}{d t} H_{1}^{(\ell)}+c\left(\left\|\partial_{x}^{\ell}\left(n-n_{\infty}\right)\right\|_{H^{1}}^{2}+\left\|\partial_{x}^{\ell} E\right\|_{L^{2}}^{2}\right) \leq \varepsilon\left\|\partial_{x}^{\ell+1} B\right\|_{L^{2}}^{2}+C_{\varepsilon}\left\|\partial_{x}^{\ell} u\right\|_{H^{1}}^{2}+G_{1}^{(\ell)}
$$

for any $\varepsilon>0$, where $H_{1}^{(\ell)}=\int_{\mathbb{R}^{3}} \mathcal{H}_{1}^{(\ell)} d x$ with $\mathcal{H}_{1}^{(\ell)}=-a_{\infty} \partial_{x}^{\ell}\left(n-n_{\infty}\right) \operatorname{div} \partial_{x}^{\ell} u+\partial_{x}^{\ell} u$. $\partial_{x}^{\ell} E$, and $C_{\varepsilon}$ is a positive constant depending on $\varepsilon$. As in [23] we have

$$
\begin{gathered}
H_{1}^{(\ell)} \leq C\left(\left\|\partial_{x}^{\ell}\left(n-n_{\infty}, E\right)\right\|_{L^{2}}+\left\|\partial_{x}^{\ell} u\right\|_{H^{1}}^{2}\right), \\
G_{1}^{(\ell)} \leq C\left\|w-w_{\infty}\right\|_{L^{\infty}}\left(\left\|\partial_{x}^{\ell}\left(n-n_{\infty}, u\right)\right\|_{H^{1}}^{2}+\left\|\partial_{x}^{\ell} E\right\|_{L^{2}}^{2}\right) \\
\quad+C\|u\|_{L^{\infty}}\left\|\partial_{x}^{\ell}\left(B-B_{\infty}\right)\right\|_{L^{2}}^{2}
\end{gathered}
$$

for $0 \leq \ell \leq s-1$, where the term $C\|u\|_{L^{\infty}}\left\|\partial_{x}^{\ell}\left(B-B_{\infty}\right)\right\|_{L^{2}}^{2}$ in the estimate of $G_{1}^{(\ell)}$ can be omitted if $\ell=0$. Here we note that the term $\varepsilon\left\|\partial_{x}^{\ell+1} B\right\|_{L^{2}}^{2}$ in (3.7) comes from $\int_{\mathbb{R}^{3}} \partial_{x}^{\ell} u \cdot \operatorname{rot} \partial_{x}^{\ell} B d x$. If we integrate it by parts, then we can modify (3.7) as

(3.9) $\frac{d}{d t} H_{1}^{(\ell)}+c\left(\left\|\partial_{x}^{\ell}\left(n-n_{\infty}\right)\right\|_{H^{1}}^{2}+\left\|\partial_{x}^{\ell} E\right\|_{L^{2}}^{2}\right) \leq \varepsilon\left\|\partial_{x}^{\ell}\left(B-B_{\infty}\right)\right\|_{L^{2}}^{2}+C_{\varepsilon}\left\|\partial_{x}^{\ell} u\right\|_{H^{1}}^{2}+G_{1}^{(\ell)}$.

Finally, we take the inner product of the third and the fourth equation of $\partial_{x}^{\ell}(3.6)$ with $-\operatorname{rot} \partial_{x}^{\ell} B$ and $-\operatorname{rot} \partial_{x}^{\ell} E$, respectively. Then we add these two equations and integrate over $x \in \mathbb{R}^{3}$. After a simple computation as in [23], using (1.3), we get

$$
\frac{d}{d t} H_{2}^{(\ell)}+c\left\|\partial_{x}^{\ell+1} B\right\|_{L^{2}}^{2} \leq C\left(\left\|\partial_{x}^{\ell+1} E\right\|_{L^{2}}^{2}+\left\|\partial_{x}^{\ell} u\right\|_{L^{2}}^{2}\right)+G_{2}^{(\ell)},
$$

where $H_{2}^{(\ell)}=-\int_{\mathbb{R}^{3}} \partial_{x}^{\ell} E \cdot \operatorname{rot} \partial_{x}^{\ell} B d x$. As in [23], we easily see that

$$
\begin{aligned}
H_{2}^{(\ell)} & \leq C\left(\left\|\partial_{x}^{\ell} E\right\|_{L^{2}}+\left\|\partial_{x}^{\ell+1} B\right\|_{L^{2}}^{2}\right), \\
G_{2}^{(\ell)} & \leq C\left\|\left(n-n_{\infty}, u\right)\right\|_{L^{\infty}}\left\|\partial_{x}^{\ell}\left(n-n_{\infty}, u\right)\right\|_{L^{2}}\left\|\partial_{x}^{\ell+1} B\right\|_{L^{2}}
\end{aligned}
$$


for $0 \leq \ell \leq s-1$. The standard energy inequality (3.4) together with (3.7), (3.9) and (3.10) was sufficient to show the uniform energy estimate of solutions to the Euler-Maxwell system (1.1), (1.2), (1.3). For the details, see [23].

4. Time weighted energy estimates. The key to the proof of our main Theorem 2.2 is to derive the following time weighted energy estimate as a priori estimate of solutions to the problem (1.1), (1.5).

Proposition 4.1 (Time weighted energy estimate). Let $s \geq 6$ and suppose that the initial data satisfy $w_{0}-w_{\infty} \in H^{s}$ and (1.6). Let $w(t, x)$ be a solution to the problem (1.1), (1.5) satisfying $w-w_{\infty} \in C\left([0, T] ; H^{s}\right) \cap C^{1}\left([0, T] ; H^{s-1}\right)$ and $(3.1)$ for some $T>0$. Then there are some positive constants $\varepsilon_{3}$ and $C$ independent of $T$ such that if $I_{0} \leq \varepsilon_{3}$, then the following a priori estimate holds for $t \in[0, T]$ :

$$
N(t)^{2}+D(t)^{2}+N_{\perp}(t)^{2} \leq C I_{0}^{2}
$$

To prove Proposition 4.1, we introduce the following norms:

$$
W_{0}(t):=\sup _{0 \leq \tau \leq t}\left\|\left(w-w_{\infty}\right)(\tau)\right\|_{W^{1, \infty}}, \quad J_{0}(t)^{2}:=\int_{0}^{t}\left\|\left(n-n_{\infty}, u\right)(\tau)\right\|_{L^{\infty}}^{2} d \tau .
$$

Note that $W_{0}(t) \leq C N_{0}(t) \leq C N(t)$ for $s \geq 3$ and $J_{0}(t) \leq C D_{0}(t) \leq C D(t)$ for $s \geq 2$, which are due to the Gagliardo-Nirenberg inequality in $\mathbb{R}^{3}:\|v\|_{L^{\infty}} \leq$ $C\|v\|_{L^{2}}^{1 / 4}\left\|\partial_{x}^{2} v\right\|_{L^{2}}^{3 / 4}$. We also use the norm

$$
W_{\perp}(t):=\sup _{0 \leq \tau \leq t}(1+\tau)\left\|\left(n-n_{\infty}, u, E\right)(\tau)\right\|_{W^{1, \infty}}
$$

which can be estimated by $N_{\perp}(t)$ in $(2.9)$ as

$$
W_{\perp}(t) \leq C N_{\perp}(t)
$$

for $s \geq 6$. Indeed, by applying the Gagliardo-Nirenberg inequality for $v=(n-$ $\left.n_{\infty}, u, E\right)$, we obtain

$$
\|v\|_{L^{\infty}} \leq C\|v\|_{L^{2}}^{1 / 4}\left\|\partial_{x}^{2} v\right\|_{L^{2}}^{3 / 4} \leq C\|v\|_{H^{s-2}}^{1 / 4}\left\|\partial_{x}^{2} v\right\|_{H^{s-6}}^{3 / 4} \leq C N_{\perp}(t)(1+t)^{-5 / 4}
$$

for $s \geq 6$. Also we have $\left\|\partial_{x} v\right\|_{L^{\infty}} \leq C\left\|\partial_{x} v\right\|_{H^{2}} \leq C\left\|\partial_{x} v\right\|_{H^{s-4}} \leq C N_{\perp}(t)(1+t)^{-1}$ for $s \geq 6$. This shows (4.2).

In order to show the a priori estimate in Proposition 4.1, we derive the following time weighted energy inequality for the system (1.1).

Proposition 4.2 (Time weighted energy inequality). Let $s \geq 3$ and assume that the same conditions in Proposition 4.1 hold true. Then we have the following time weighted energy inequality:

$$
N(t)^{2}+D(t)^{2} \leq C I_{0}^{2}+C\left(N(t)+W_{\perp}(t)\right) D(t)^{2} .
$$

The proof of Proposition 4.2 consists of two lemmas. The first lemma is on the time weighted energy norm $N(t)$. 
Lemma 4.3. Let $s \geq 3$. Under the same conditions in Proposition 4.2, we have the following time weighted energy inequality:

$$
\begin{aligned}
& (1+t)^{k}\left\|\partial_{x}^{k}\left(w-w_{\infty}\right)(t)\right\|_{H^{s-2 k}}^{2}+\int_{0}^{t}(1+\tau)^{k}\left\|\partial_{x}^{k} u(\tau)\right\|_{H^{s-2 k}}^{2} d \tau \\
& \leq C I_{0}^{2}+k C \int_{0}^{t}(1+\tau)^{k-1}\left\|\partial_{x}^{k}\left(w-w_{\infty}\right)(\tau)\right\|_{H^{s-2 k}}^{2} d \tau+C\left(N(t)+W_{\perp}(t)\right) D(t)^{2}
\end{aligned}
$$

for $0 \leq k \leq[s / 2]$.

Proof. Let $0 \leq k \leq[s / 2]$. Multiplying (3.4) by $(1+t)^{k}$, integrating the resultant inequality with respect to $t$, and summing up for $\ell$ with $k \leq \ell \leq s-k$, we obtain

$$
\begin{gathered}
(1+t)^{k}\left\|\partial_{x}^{k}\left(w-w_{\infty}\right)(t)\right\|_{H^{s-2 k}}^{2}+\int_{0}^{t}(1+\tau)^{k}\left\|\partial_{x}^{k} u(\tau)\right\|_{H^{s-2 k}}^{2} d \tau \\
\leq C I_{0}^{2}+k C \int_{0}^{t}(1+\tau)^{k-1}\left\|\partial_{x}^{k}\left(w-w_{\infty}\right)(\tau)\right\|_{H^{s-2 k}}^{2} d \tau \\
+C \int_{0}^{t}(1+\tau)^{k} \sum_{\ell=k}^{s-k}\left(R_{A}^{(\ell)}+R_{L}^{(\ell)}\right)(\tau) d \tau
\end{gathered}
$$

where we used the estimate $(3.5)$ for $H_{0}^{(\ell)}$.

We need to estimate the remainder terms in (4.5) by using the dissipation norm $D(t)$ in (2.7). To this end, we observe that $\left\|\partial_{x}^{k}\left(w-w_{\infty}\right)\right\|_{H^{s-2 k}}^{2} \leq \tilde{D}_{k-1}^{2}$ (for $\left.k \geq 1\right)$, which together with the definition of $D(t)$ gives

$$
\int_{0}^{t}(1+\tau)^{k-1}\left\|\partial_{x}^{k}\left(w-w_{\infty}\right)(\tau)\right\|_{H^{s-2 k}}^{2} d \tau \leq \int_{0}^{t}(1+\tau)^{k-1} \tilde{D}_{k-1}(\tau)^{2} d \tau \leq D(t)^{2}
$$

for $1 \leq k \leq[s / 2]$. Now we estimate the remainder term including $R_{A}^{(\ell)}$. When $1 \leq k \leq[s / 2]$, by using (3.5) and (4.6), we have

$$
\int_{0}^{t}(1+\tau)^{k} \sum_{\ell=k}^{s-k} R_{A}^{(\ell)}(\tau) d \tau \leq C \int_{0}^{t}(1+\tau)^{k}\left\|\partial_{x}(n, u)\right\|_{L^{\infty}}\left\|\partial_{x}^{k}(n, u)\right\|_{H^{s-2 k}}^{2} d \tau \leq C W_{\perp}(t) D(t)^{2} .
$$

On the other hand, when $k=0$, we can estimate the term by using (3.5) as

$$
\begin{aligned}
\int_{0}^{t} \sum_{\ell=0}^{s} R_{A}^{(\ell)}(\tau) d \tau & \leq C \int_{0}^{t}\left\|\partial_{x}(n, u)\right\|_{L^{\infty}}\left\|\left(n-n_{\infty}, u\right)\right\|_{H^{s}}^{2} d \tau \\
& \leq C W_{0}(t) D_{0}(t)^{2} \leq C N(t) D(t)^{2} .
\end{aligned}
$$

Finally, we estimate the term including $R_{L}^{(\ell)}$. When $1 \leq k \leq[s / 2]$, by using $(3.5)$ and (4.6), we get

$$
\begin{aligned}
\int_{0}^{t}(1+\tau)^{k} & \sum_{\ell=k}^{s-k} R_{L}^{(\ell)}(\tau) d \tau \leq C \int_{0}^{t}(1+\tau)^{k}\left\|B-B_{\infty}\right\|_{L^{\infty}}\left\|\partial_{x}^{k} u\right\|_{H^{s-2 k}}^{2} d \tau \\
& +C \int_{0}^{t}(1+\tau)^{k}\left\|\left(n-n_{\infty}, u\right)\right\|_{L^{\infty}}\left\|\partial_{x}^{k}(n, u)\right\|_{H^{s-2 k}}\left\|\partial_{x}^{k}(E, B)\right\|_{H^{s-2 k}} d \tau \\
& \leq C W_{0}(t) D(t)^{2}+C W_{\perp}(t) D(t)^{2} \leq C\left(N(t)+W_{\perp}(t)\right) D(t)^{2}
\end{aligned}
$$


Also, when $k=0$, we estimate the term as

$$
\begin{aligned}
\int_{0}^{t} \sum_{\ell=0}^{s} R_{L}^{(\ell)}(\tau) d \tau & =\int_{0}^{t} \sum_{\ell=1}^{s} R_{L}^{(\ell)}(\tau) d \tau \leq C \int_{0}^{t}\left\|B-B_{\infty}\right\|_{L^{\infty}}\left\|\partial_{x} u\right\|_{H^{s-1}}^{2} d \tau \\
& +C \int_{0}^{t}\left\|\left(n-n_{\infty}, u\right)\right\|_{L^{\infty}}\left\|\partial_{x}(n, u)\right\|_{H^{s-1}}\left\|\partial_{x}(E, B)\right\|_{H^{s-1}} d \tau \\
& \leq C W_{0}(t) D_{0}(t)^{2}+C N_{0}(t) J_{0}(t) D_{0}(t) \leq C N(t) D(t)^{2} .
\end{aligned}
$$

Substituting these estimates into (4.5), we arrive at the desired estimate (4.4). This completes the proof of Lemma 4.3 .

The second lemma is on the time weighted dissipation norm $D(t)$.

Lemma 4.4. Let $s \geq 3$. Under the same conditions in Proposition 4.2, we have the following time weighted energy inequality:

$$
\begin{aligned}
\int_{0}^{t}(1+\tau)^{k} \tilde{D}_{k}(\tau)^{2} d \tau \leq C I_{0}^{2}+ & k C \int_{0}^{t}(1+\tau)^{k-1}\left\|\partial_{x}^{k}\left(w-w_{\infty}\right)(\tau)\right\|_{H^{s-2 k}}^{2} d \tau \\
& +C\left(N(t)+W_{\perp}(t)\right) D(t)^{2}
\end{aligned}
$$

for $0 \leq k \leq[s / 2]-1$, where $\tilde{D}_{k}$ is given in (2.8).

Proof. First, we multiply (3.7) with $\ell=k$ by $(1+t)^{k}$ and integrate with respect to $t$. Also, we multiply $(3.9)$ by $(1+t)^{k}$, sum up the resultant inequality for $\ell$ with $k+1 \leq \ell \leq s-k-1$ and integrate with respect to $t$. Then, adding these two inequalities, we obtain

$$
\begin{aligned}
& \int_{0}^{t}(1+\tau)^{k}\left(\left\|\partial_{x}^{k}\left(n-n_{\infty}\right)(\tau)\right\|_{H^{s-2 k}}^{2}+\left\|\partial_{x}^{k} E(\tau)\right\|_{H^{s-2 k-1}}^{2}\right) d \tau \\
& \leq C I_{0}^{2}+\varepsilon \int_{0}^{t}(1+\tau)^{k}\left\|\partial_{x}^{k+1} B(\tau)\right\|_{H^{s-2 k-2}}^{2} d \tau \\
& +C(1+t)^{k}\left\|\partial_{x}^{k}\left(w-w_{\infty}\right)(t)\right\|_{H^{s-2 k}}^{2}+C_{\varepsilon} \int_{0}^{t}(1+\tau)^{k}\left\|\partial_{x}^{k} u(\tau)\right\|_{H^{s-2 k}}^{2} d \tau \\
& +k C \int_{0}^{t}(1+\tau)^{k-1}\left\|\partial_{x}^{k}\left(w-w_{\infty}\right)(\tau)\right\|_{H^{s-2 k}}^{2} d \tau+C \int_{0}^{t}(1+\tau)^{k} \sum_{\ell=k}^{s-k-1} G_{1}^{(\ell)}(\tau) d \tau
\end{aligned}
$$

for any $\varepsilon>0$, where $0 \leq k \leq[s / 2]-1$, and $C_{\varepsilon}$ is a positive constant depend on $\varepsilon$. Here we used the estimate $(3.8)$ for $H_{1}^{(\ell)}$. The remainder term in (4.8) is estimated as follows. When $1 \leq k \leq[s / 2]-1$, by using (3.8) and (4.6), we have

$$
\begin{aligned}
& \int_{0}^{t}(1+\tau)^{k} \sum_{\ell=k}^{s-k-1} G_{1}^{(\ell)}(\tau) d \tau \\
& \leq C \int_{0}^{t}(1+\tau)^{k}\left\|w-w_{\infty}\right\|_{L^{\infty}}\left(\left\|\partial_{x}^{k}\left(n-n_{\infty}, u\right)\right\|_{H^{s-2 k}}+\left\|\partial_{x}^{k} E\right\|_{H^{s-2 k-1}}^{2}\right) d \tau \\
& +C \int_{0}^{t}(1+\tau)^{k}\|u\|_{L^{\infty}}\left\|\partial_{x}^{k}\left(B-B_{\infty}\right)\right\|_{H^{s-2 k-1}} d \tau \\
& \leq C W_{0}(t) D(t)^{2}+C W_{\perp}(t) D(t)^{2} \leq C\left(N(t)+W_{\perp}(t)\right) D(t)^{2} .
\end{aligned}
$$


On the other hand, when $k=0$, we estimate the term simply as

$$
\begin{aligned}
\int_{0}^{t} \sum_{\ell=0}^{s-1} G_{1}^{(\ell)}(\tau) d \tau & \leq C \int_{0}^{t}\left\|w-w_{\infty}\right\|_{L^{\infty}}\left(\left\|\left(n-n_{\infty}, u\right)\right\|_{H^{s}}+\|E\|_{H^{s-1}}^{2}+\left\|\partial_{x} B\right\|_{H^{s-2}}\right) d \tau \\
& \leq C W_{0}(t) D_{0}(t)^{2} \leq C N(t) D(t)^{2}
\end{aligned}
$$

Next, we multiply $(3.10)$ by $(1+t)^{k}$, sum up the resultant inequality for $\ell$ with $k \leq \ell \leq s-k-2$ and integrating with respect $t$. This yields

$$
\begin{aligned}
& \int_{0}^{t}(1+\tau)^{k}\left\|\partial_{x}^{k+1} B(\tau)\right\|_{H^{s-2 k-2}}^{2} d \tau \leq C I_{0}^{2}+C \int_{0}^{t}(1+\tau)^{k}\left\|\partial_{x}^{k+1} E(\tau)\right\|_{H^{s-2 k-2}}^{2} d \tau \\
& +C(1+t)^{k}\left\|\partial_{x}^{k}\left(w-w_{\infty}\right)(t)\right\|_{H^{s-2 k-1}}^{2}+C \int_{0}^{t}(1+\tau)^{k}\left\|\partial_{x}^{k} u(\tau)\right\|_{H^{s-2 k-2}}^{2} d \tau \\
& +k C \int_{0}^{t}(1+\tau)^{k-1}\left\|\partial_{x}^{k}\left(w-w_{\infty}\right)(\tau)\right\|_{H^{s-2 k-1}}^{2} d \tau+C \int_{0}^{t}(1+\tau)^{k} \sum_{\ell=k}^{s-k-2} G_{2}^{(\ell)}(\tau) d \tau
\end{aligned}
$$

for $0 \leq k \leq[s / 2]-1$, where we used the estimate (3.11) for $H_{2}^{(\ell)}$. The remainder term in (4.9) is estimated by using (3.11) as

$$
\begin{aligned}
& \int_{0}^{t}(1+\tau)^{k} \sum_{\ell=k}^{s-k-2} G_{2}^{(\ell)}(\tau) d \tau \\
& \leq C \int_{0}^{t}(1+\tau)^{k}\left\|\left(n-n_{\infty}, u\right)\right\|_{L^{\infty}}\left\|\partial_{x}^{k}\left(n-n_{\infty}, u\right)\right\|_{H^{s-2 k-2}}\left\|\partial_{x}^{k+1} B\right\|_{H^{s-2 k-2}} d \tau \\
& \leq C W_{0}(t) D(t)^{2} \leq C N(t) D(t)^{2} .
\end{aligned}
$$

Finally, we combine (4.8) and (4.9), and take $\varepsilon>0$ sufficiently small. This yields

$$
\begin{aligned}
& \int_{0}^{t}(1+\tau)^{k}\left(\left\|\partial_{x}^{k}\left(n-n_{\infty}\right)(\tau)\right\|_{H^{s-2 k}}^{2}+\left\|\partial_{x}^{k} E(\tau)\right\|_{H^{s-2 k+1}}^{2}+\left\|\partial_{x}^{k+1} B(\tau)\right\|_{H^{s-2 k-2}}^{2}\right) d \tau \\
& \leq C I_{0}^{2}+C(1+t)^{k}\left\|\partial_{x}^{k}\left(w-w_{\infty}\right)(t)\right\|_{H^{s-2 k}}^{2}+C \int_{0}^{t}(1+\tau)^{k}\left\|\partial_{x}^{k} u(\tau)\right\|_{H^{s-2 k}}^{2} d \tau \\
& +k C \int_{0}^{t}(1+\tau)^{k-1}\left\|\partial_{x}^{k}\left(w-w_{\infty}\right)(\tau)\right\|_{H^{s-2 k}}^{2} d \tau+C\left(N(t)+W_{\perp}(t)\right) D(t)^{2}
\end{aligned}
$$

for $0 \leq k \leq[s / 2]-1$, which together with (4.4) gives the desired estimate (4.7). This completes the proof of Lemma 4.4 .

Proof of Proposition 4.2. For the proof of Proposition 4.2, it suffices to show the following estimates:

$$
\begin{aligned}
& (1+t)^{k}\left\|\partial_{x}^{k}\left(w-w_{\infty}\right)(t)\right\|_{H^{s-2 k}}^{2}+\int_{0}^{t}(1+\tau)^{k}\left\|\partial_{x}^{k} u(\tau)\right\|_{H^{s-2 k}}^{2} d \tau \\
& \leq C I_{0}^{2}+C\left(N(t)+W_{\perp}(t)\right) D(t)^{2} \\
& \int_{0}^{t}(1+\tau)^{k} \tilde{D}_{k}(\tau)^{2} d \tau \leq C I_{0}^{2}+C\left(N(t)+W_{\perp}(t)\right) D(t)^{2}
\end{aligned}
$$


where $0 \leq k \leq[s / 2]$ in (4.10), $0 \leq k \leq[s / 2]-1$ in (4.11), and $\tilde{D}_{k}$ is given in (2.8). These estimates can be obtained by applying the induction argument with respect to $k$ to the inequalities (4.4) and (4.7), respectively. Indeed, putting $k=0$ in (4.4) and (4.7), we see that (4.10) and (4.11) hold true for $k=0$. Also, we put $k=1$ in (4.4) and apply (4.6) with $k=1$ and (4.11) with $k=0$. This shows that (4.10) hold true also for $k=1$. Thus we have shown the estimates (4.10) and (4.11) for $s=3$. When $s \geq 4$, letting $1 \leq j \leq[s / 2]-1$, we assume that (4.11) holds true for $k=j-1$. Then we show (4.10) for $k=j, j+1$ and (4.11) for $k=j$. To this end, we put $k=j$ in (4.4) and (4.7), and apply (4.6) with $k=j$ and (4.11) with $k=j-1$. This gives (4.10) and (4.11) for $k=j$. Moreover, letting $k=j+1$ in (4.4) and using (4.6) with $k=j+1$ and (4.11) with $k=j$, we find that (4.10) holds true also for $k=j+1$. Thus we have shown (4.10) and (4.11) also for $s \geq 4$. This completes the proof of Proposition 4.2 .

In order to get the desired a priori estimate (4.1) in Proposition 4.1, we need to control the quantity $N_{\perp}(t)$ (cf. (4.2)).

Proposition 4.5. Let $s \geq 3$. Under the same conditions in Proposition 4.2, we have the following time weighted inequality:

$$
N_{\perp}(t) \leq C I_{0}+C N(t)+C N(t)\left(N_{\perp}(t)+W_{\perp}(t)\right) .
$$

Proof. We rewrite the second and the third equations of (3.6) as

$$
\left\{\begin{array}{l}
u_{t}+E+u \times B_{\infty}+u=\tilde{g}_{2}, \\
E_{t}-n_{\infty} u=\tilde{g}_{3},
\end{array}\right.
$$

where

$$
\begin{aligned}
& \tilde{g}_{2}=-(u \cdot \nabla) u-a(n) \nabla n-u \times\left(B-B_{\infty}\right), \\
& \tilde{g}_{3}=\operatorname{rot} B+\left(n-n_{\infty}\right) u .
\end{aligned}
$$

We regard (4.13) as a system of ordinary differential equations for $(u, E)$ and write as

$$
\left(\begin{array}{cc}
n_{\infty} I & O \\
O & I
\end{array}\right)\left(\begin{array}{l}
u \\
E
\end{array}\right)_{t}+\left(\begin{array}{cc}
n_{\infty}\left(I-\Omega_{B_{\infty}}\right) & n_{\infty} I \\
-n_{\infty} I & O
\end{array}\right)\left(\begin{array}{l}
u \\
E
\end{array}\right)=\left(\begin{array}{c}
n_{\infty} \tilde{g}_{2} \\
\tilde{g}_{3}
\end{array}\right)
$$

We see that the fundamental solution of (4.14) decays exponentially for $t \rightarrow \infty$. Therefore, solving (4.14) by using the fundamental solution, applying $\partial_{x}^{\ell}$ to the resultant solution formula and taking the $L^{2}$-norm, we obtain

$$
\left\|\partial_{x}^{\ell}(u, E)(t)\right\|_{L^{2}} \leq C I_{0} e^{-c t}+C \int_{0}^{t} e^{-c(t-\tau)}\left\|\partial_{x}^{\ell}\left(\tilde{g}_{2}, \tilde{g}_{3}\right)(\tau)\right\|_{L^{2}} d \tau,
$$

where $0 \leq \ell \leq[s / 2]-1$. Here we see that

$$
\begin{aligned}
& \left\|\partial_{x}^{\ell}\left(\tilde{g}_{2}, \tilde{g}_{3}\right)\right\|_{L^{2}} \leq C\left\|\partial_{x}^{\ell+1}(n, u, B)\right\|_{L^{2}} \\
& \quad+C\left\|w-w_{\infty}\right\|_{L^{\infty}}\left\|\partial_{x}^{\ell}\left(n-n_{\infty}, u\right)\right\|_{L^{2}}+C\|u\|_{L^{\infty}}\left\|\partial_{x}^{\ell}\left(B-B_{\infty}\right)\right\|_{L^{2}} .
\end{aligned}
$$


Therefore, summing up (4.15) for $\ell$ with $k \leq \ell \leq s-k-2$ and using (4.16), we obtain

$$
\begin{aligned}
\left\|\partial_{x}^{k}(u, E)(t)\right\|_{H^{s-2 k-2}} \leq C I_{0} e^{-c t}+C \int_{0}^{t} e^{-c(t-\tau)}\left\|\partial_{x}^{k+1} w(\tau)\right\|_{H^{s-2 k-2}} d \tau \\
+C \int_{0}^{t} e^{-c(t-\tau)}\left\|\left(w-w_{\infty}\right)(\tau)\right\|_{L^{\infty}}\left\|\partial_{x}^{k}\left(n-n_{\infty}, u\right)(\tau)\right\|_{H^{s-2 k-2}} d \tau \\
+C \int_{0}^{t} e^{-c(t-\tau)}\|u(\tau)\|_{L^{\infty}}\left\|\partial_{x}^{k}\left(B-B_{\infty}\right)(\tau)\right\|_{H^{s-2 k-2}} d \tau
\end{aligned}
$$

where $0 \leq k \leq[s / 2]-1$. We denote the integrals on the right hand side of (4.17) by $K_{1}, K_{2}$ and $K_{3}$, respectively. These integrals can be estimated as follows.

$$
\begin{gathered}
K_{1} \leq C N(t) \int_{0}^{t} e^{-c(t-\tau)}(1+\tau)^{-\frac{k+1}{2}} d \tau \leq C N(t)(1+t)^{-\frac{k+1}{2}} \\
K_{2} \leq C W_{0}(t) N_{\perp}(t) \int_{0}^{t} e^{-c(t-\tau)}(1+\tau)^{-\frac{k+1}{2}} d \tau \leq C N(t) N_{\perp}(t)(1+t)^{-\frac{k+1}{2}} \\
K_{3} \leq C W_{\perp}(t) N(t) \int_{0}^{t} e^{-c(t-\tau)}(1+\tau)^{-1-\frac{k}{2}} d \tau \leq C W_{\perp}(t) N(t)(1+t)^{-1-\frac{k}{2}} .
\end{gathered}
$$

Substituting these estimates into (4.17), we get

$$
(1+t)^{\frac{k+1}{2}}\left\|\partial_{x}^{k}(u, E)(t)\right\|_{H^{s-2 k-2}} \leq C I_{0}+C N(t)+C N(t)\left(N_{\perp}(t)+W_{\perp}(t)\right),
$$

where $0 \leq k \leq[s / 2]-1$. On the other hand, using (1.2), we see that $\| \partial_{x}^{k}(n-$ $\left.n_{\infty}\right)(t)\left\|_{H^{s-2 k-2}} \leq\right\| \partial_{x}^{k+1} E(t) \|_{H^{s-2 k-2}} \leq N(t)(1+t)^{-(k+1) / 2}$. Namely, we have

$$
(1+t)^{\frac{k+1}{2}}\left\|\partial_{x}^{k}\left(n-n_{\infty}\right)(t)\right\|_{H^{s-2 k-2}} \leq N(t),
$$

where $0 \leq k \leq[s / 2]-1$. The desired inequality (4.12) directly follows from (4.18) and (4.19). This completes the proof of Lemma 4.5.

Proof of Proposition 4.1. Let $s \geq 6$. Then we have (4.2). Substituting (4.2) in (4.3) and (4.12), we obtain

$$
\begin{gathered}
N(t)^{2}+D(t)^{2} \leq C I_{0}^{2}+C\left(N(t)+N_{\perp}(t)\right) D(t)^{2}, \\
N_{\perp}(t) \leq C I_{0}^{2}+C N(t)+C N(t) N_{\perp}(t) .
\end{gathered}
$$

Put $Y(t)=N(t)+D(t)+N_{\perp}(t)$. Then we obtain the inequality $Y(t)^{2} \leq C I_{0}^{2}+C Y(t)^{3}$, from which we can deduce that $Y(t) \leq C I_{0}$, provided that $I_{0}$ is suitably small, say, $I_{0} \leq \varepsilon_{3}$. This gives the desired estimate (4.1) and hence the proof of Proposition 4.1 is complete.

Proof of Theorem 2.2. The global existence of our solution can be proved by the standard continuation argument based on a local existence result and the a priori estimate given in Proposition 4.1. This global solution verifies the time weighted energy estimate (2.10) for all $t \geq 0$. Consequently, we we have the $L^{2}$ decay estimates (2.11) and (2.12). Moreover, using (1.2) and (2.12), we obtain

$$
\left\|\partial_{x}^{k}\left(n-n_{\infty}\right)(t)\right\|_{H^{s-2 k-4}} \leq\left\|\partial_{x}^{k+1} E(t)\right\|_{H^{s-2 k-4}} \leq C I_{0}(1+t)^{-1-k / 2}
$$

for $0 \leq k \leq[s / 2]-2$. Thus the proof of Theorem 2.2 is complete. 
5. Decay estimate (linear case). In this section, we derive the optimal decay estimate for the linearized Euler-Maxwell system. First we introduce $v=n u / n_{\infty}$ and rewrite (1.1) as

$$
\left\{\begin{array}{l}
n_{t}+n_{\infty} \operatorname{div} v=0 \\
v_{t}+a_{\infty} \nabla n+E+v \times B_{\infty}+v=\left(\operatorname{div} Q_{2}+r_{2}\right) / n_{\infty} \\
E_{t}-\operatorname{rot} B-n_{\infty} v=0 \\
B_{t}+\operatorname{rot} E=0
\end{array}\right.
$$

where $a_{\infty}=a\left(n_{\infty}\right)$ with $a(n)=p^{\prime}(n) / n$ and

$$
\begin{aligned}
Q_{2} & =-n u \otimes u-\left\{p(n)-p\left(n_{\infty}\right)-p^{\prime}\left(n_{\infty}\right)\left(n-n_{\infty}\right)\right\} I, \\
r_{2} & =-\left(n-n_{\infty}\right) E-n u \times\left(B-B_{\infty}\right) .
\end{aligned}
$$

We put $z=\left(n-n_{\infty}, v, E, B-B_{\infty}\right)^{T}$ and rewrite (5.1) in the vector form as

$$
A^{0} z_{t}+\sum_{j=1}^{3} A^{j} z_{x_{j}}+L z=\sum_{j=1}^{3} q_{x_{j}}^{j}+r
$$

where $A^{0}, A^{j}$ and $L$ are the constant matrices given by (2.3) with $w=w_{\infty}$, and $q^{j}=\left(0, q_{2}^{j}, 0,0\right)^{T}$ and $r=\left(0, r_{2}, 0,0\right)^{T}$; the $i$ component of the vector $q_{2}^{j}$ is given by the $(i, j)$ component of the matrix $Q_{2}$. We note that $q^{j}, r=O\left(\left|w-w_{\infty}\right|^{2}\right)$.

We consider the linearized Euler-Maxwell system

$$
A^{0} z_{t}+\sum_{j=1}^{3} A^{j} z_{x_{j}}+L z=0 .
$$

We write $z=(\rho, v, E, h)^{T}$, where $\rho=n-n_{\infty}$ and $h=B-B_{\infty}$. Then the system (5.3) is written explicitly as

$$
\left\{\begin{array}{l}
\rho_{t}+n_{\infty} \operatorname{div} v=0 \\
v_{t}+a_{\infty} \nabla \rho+E+v \times B_{\infty}+v=0 \\
E_{t}-\operatorname{rot} h-n_{\infty} v=0 \\
h_{t}+\operatorname{rot} E=0
\end{array}\right.
$$

The equations (1.2) and (1.3), which are linear, are reduced to

$$
\operatorname{div} E=-\rho,
$$

$$
\operatorname{div} h=0 .
$$

Let $z_{0}=\left(\rho_{0}, v_{0}, E_{0}, h_{0}\right)^{T}$ be the initial data for $z$ and assume that

$$
\operatorname{div} E_{0}=-\rho_{0}, \quad \operatorname{div} h_{0}=0 .
$$

Then, as remarked in Section 1, the corresponding solution of (5.4) always satisfies (5.5) and (5.6) for all $t>0$. 
We apply the Fourier transform of (5.3), obtaining

$$
A^{0} \hat{z}_{t}+i|\xi| A(\omega) \hat{z}+L \hat{z}=0
$$

where $A(\omega)=\sum_{j=1}^{3} A^{j} \omega_{j}$ and $\omega=\xi /|\xi| \in S^{2}$. Put

$$
\hat{\Phi}(i \xi)=-\left(A^{0}\right)^{-1}(i|\xi| A(\omega)+L) .
$$

Then the solution of (5.8) with the initial data $\hat{z}(0, \xi)=\hat{z}_{0}(\xi)$ is given by $\hat{z}(t, \xi)=$ $e^{t \hat{\Phi}(i \xi)} \hat{z}_{0}(\xi)$. The semigroup $e^{t \Phi}$ associated with the linearized Euler-Maxwell system (5.3) is then defined by the formula

$$
\left(e^{t \Phi} \varphi\right)(x)=\mathcal{F}^{-1}\left[e^{t \hat{\Phi}(i \xi)} \hat{\varphi}(\xi)\right](x) .
$$

We have the following pointwise estimate for the matrix exponential $e^{t \hat{\Phi}(i \xi)}$.

THEOREM 5.1. Let $\hat{\Phi}(i \xi)$ be the matrix in (5.9). Let $\varphi=\left(\rho_{0}, v_{0}, E_{0}, h_{0}\right)^{T}$ be a function satisfying (5.7). Then the matrix exponential $e^{t \hat{\Phi}(i \xi)}$ applied to such a vector $\hat{\varphi}(\xi)$ verifies the pointwise estimate

$$
\left|e^{t \hat{\Phi}(i \xi)}\right| \leq C e^{-c \eta(\xi) t}
$$

for $t \geq 0$ and $\xi \in \mathbb{R}^{3}$, where $\eta(\xi)=|\xi|^{2} /\left(1+|\xi|^{2}\right)^{2}$.

As an easy consequence of (5.11), we have the following decay estimate for the semigroup $e^{t \Phi}$.

COROLlARY 5.2. Let $e^{t \Phi}$ be the semigroup associated with the linearized EulerMaxwell system (5.3), which is defined in (5.10). Let $\varphi=\left(\rho_{0}, v_{0}, E_{0}, h_{0}\right)^{T}$ satisfy (5.7). Then we have the decay estimate

$$
\left\|\partial_{x}^{k} e^{t \Phi} \varphi\right\|_{L^{2}} \leq C(1+t)^{-3 / 4-k / 2}\|\varphi\|_{L^{1}}+C(1+t)^{-\ell / 2}\left\|\partial_{x}^{k+\ell} \varphi\right\|_{L^{2}},
$$

where $k$ and $\ell$ are non-negative integers.

REMARK. The decay estimate (5.12) is of the regularity-loss type because we have quantitative decay $(1+t)^{-\ell / 2}$ only by assuming the additional $\ell$-th order regularity on the initial data.

Proof of Theorem 5.1. We prove (5.11) by applying the energy method in the Fourier space. The system (5.8) is written explicitly as

$$
\begin{gathered}
\hat{\rho}_{t}+n_{\infty} i|\xi| \hat{v} \cdot \omega=0, \\
\hat{v}_{t}+a_{\infty} i|\xi| \hat{\rho} \omega+\hat{E}+\hat{v} \times B_{\infty}+\hat{v}=0, \\
\hat{E}_{t}+i|\xi| \hat{h} \times \omega-n_{\infty} \hat{v}=0, \\
\hat{h}_{t}-i|\xi| \hat{E} \times \omega=0 .
\end{gathered}
$$

Also, applying the Fourier transform to (5.5) and (5.6), we have

$$
\begin{gathered}
i|\xi| \hat{E} \cdot \omega=-\hat{\rho}, \\
i|\xi| \hat{h} \cdot \omega=0 .
\end{gathered}
$$


We construct a Lyapunov function for the system (5.13) with (5.14) and (5.15). For this purpose, we first take the inner product in $\mathbb{C}^{10}$ of $(5.8)$ with $\hat{z}$. From the real part we have

$$
\left(\hat{H}_{0}\right)_{t}+c_{0}|\hat{v}|^{2}=0
$$

where we put $\hat{H}_{0}=\left\langle A^{0} \hat{z}, \hat{z}\right\rangle=a_{\infty}|\hat{\rho}|^{2}+n_{\infty}|\hat{v}|^{2}+|\hat{E}|^{2}+|\hat{h}|^{2}$ and $c_{0}=2 n_{\infty}$. Here $\langle\cdot, \cdot\rangle$ denotes the inner product in $\mathbb{C}^{10}$. It is clear that there are positive constants $c_{1}$ and $C_{1}$ such that

$$
c_{1}|\hat{z}|^{2} \leq \hat{H}_{0} \leq C_{1}|\hat{z}|^{2} .
$$

Next we create the dissipation terms. To this end, we first multiply (5.13a) by $\left\langle a_{\infty} i|\xi| \omega \mid \hat{v}\right\rangle=a_{\infty} i|\xi| \overline{\hat{v}} \cdot \omega$, where $\langle\cdot \mid \cdot\rangle$ denotes the inner product in $\mathbb{C}^{3}$. Also, we take the inner product in $\mathbb{C}^{3}$ of $(5.13 \mathrm{~b})$ and (5.13c) with $a_{\infty} i|\xi| \hat{\rho} \omega+\hat{E}$ and $\hat{v}$, respectively. Then, adding the resultant three equalities, we obtain

$$
\begin{aligned}
& \left\{\left\langle a_{\infty} i|\xi| \hat{\rho}_{t} \omega \mid \hat{v}\right\rangle+\left\langle\hat{v}_{t}\left|a_{\infty} i\right| \xi \mid \hat{\rho} \omega\right\rangle\right\}+\left\{\left\langle\hat{v}_{t} \mid \hat{E}\right\rangle+\left\langle\hat{E}_{t} \mid \hat{v}\right\rangle\right\} \\
& +\left|a_{\infty} i\right| \xi|\hat{\rho} \omega+\hat{E}|^{2}-n_{\infty}|\hat{v}|^{2}-p_{\infty}^{\prime}|\xi|^{2}|\hat{v} \cdot \omega|^{2} \\
& +\left\langle\hat{v} \times B_{\infty}+\hat{v}\left|a_{\infty} i\right| \xi \mid \hat{\rho} \omega+\hat{E}\right\rangle+i|\xi|\langle\hat{h} \times \omega \mid \hat{v}\rangle=0
\end{aligned}
$$

where $p_{\infty}^{\prime}=p^{\prime}\left(n_{\infty}\right)$. Here we can compute the term $\left|a_{\infty} i\right| \xi|\hat{\rho} \omega+\hat{E}|^{2}$ as

$$
\begin{aligned}
\left|a_{\infty} i\right| \xi|\hat{\rho} \omega+\hat{E}|^{2} & =a_{\infty}^{2}|\xi|^{2}|\hat{\rho}|^{2}+|\hat{E}|^{2}+2 \operatorname{Re}\left\langle a_{\infty} i|\xi| \hat{\rho} \omega \mid \hat{E}\right\rangle \\
& =a_{\infty}^{2}|\xi|^{2}|\hat{\rho}|^{2}+|\hat{E}|^{2}+2 a_{\infty}|\hat{\rho}|^{2}
\end{aligned}
$$

where we used (5.14) in the last equality. Thus, from the real part of (5.17), we obtain

$$
\left(\hat{H}_{1}\right)_{t}+\hat{D}_{1}=\hat{M}_{1}
$$

where $\hat{H}_{1}, \hat{D}_{1}$ and $\hat{M}_{1}$ are given by

$$
\begin{aligned}
& \hat{H}_{1}=\operatorname{Re}\left\{a_{\infty} i|\xi|\langle\hat{\rho} \omega \mid \hat{v}\rangle+\langle\hat{v} \mid \hat{E}\rangle\right\}, \quad \hat{D}_{1}=2 a_{\infty}|\hat{\rho}|^{2}+a_{\infty}^{2}|\xi|^{2}|\hat{\rho}|^{2}+|\hat{E}|^{2}, \\
& \hat{M}_{1}=-\operatorname{Re}\left\{i|\xi|\langle\hat{h} \times \omega \mid \hat{v}\rangle+\left\langle\hat{v} \times B_{\infty}+\hat{v}\left|a_{\infty} i\right| \xi \mid \hat{\rho} \omega+\hat{E}\right\rangle\right\}+n_{\infty}|\hat{v}|^{2}+p_{\infty}^{\prime}|\xi|^{2}|\hat{v} \cdot \omega|^{2} .
\end{aligned}
$$

We can estimate the term $\hat{M}_{1}$ as

$$
\begin{aligned}
\hat{M}_{1} & \leq C|\xi||\hat{v}||\hat{h}|+C|\hat{v}|(|\xi||\hat{\rho}|+|\hat{E}|)\}+C\left(1+|\xi|^{2}\right)|\hat{v}|^{2} . \\
& \leq \varepsilon \frac{|\xi|^{2}}{1+|\xi|^{2}}|\hat{h}|^{2}+\delta\left(|\xi|^{2}|\hat{\rho}|^{2}+|\hat{E}|^{2}\right)+C_{\varepsilon, \delta}\left(1+|\xi|^{2}\right)|\hat{v}|^{2}
\end{aligned}
$$

for any $\varepsilon, \delta>0$, where $C_{\varepsilon, \delta}$ is a positive constant depends on $(\varepsilon, \delta)$. We substitute this estimate into (5.18) and choose $\delta>0$ suitably small. This yields

$$
\left(\hat{H}_{1}\right)_{t}+c_{2}\left(1+|\xi|^{2}\right)|\hat{\rho}|^{2}+c_{2}|\hat{E}|^{2} \leq \varepsilon \frac{|\xi|^{2}}{1+|\xi|^{2}}|\hat{h}|^{2}+C_{\varepsilon}\left(1+|\xi|^{2}\right)|\hat{v}|^{2}
$$

where $c_{2}$ and $C_{\varepsilon}$ are positive constants; $C_{\varepsilon}$ depends on $\varepsilon$.

Next we take the inner product in $\mathbb{C}^{3}$ of $(5.13 \mathrm{c})$ and $(5.13 \mathrm{~d})$ with $i|\xi| \hat{h} \times \omega$ and $i|\xi| \hat{E} \times \omega$, respectively. Adding the resultant two equations, we have

$$
|\xi|\left\{\left\langle\hat{E}_{t} \mid \hat{h} \times i \omega\right\rangle+\left\langle\hat{h}_{t} \mid \hat{E} \times i \omega\right\rangle\right\}+|\xi|^{2}|\hat{h} \times \omega|^{2}=|\xi|^{2}|\hat{E} \times \omega|^{2}-n_{\infty} i|\xi|\langle\hat{v} \mid \hat{h} \times \omega\rangle .
$$


Taking the real part, we get

$$
\left(|\xi| \hat{H}_{2}\right)_{t}+|\xi|^{2}|\hat{h} \times \omega|^{2}=|\xi|^{2}|\hat{E} \times \omega|^{2}-\operatorname{Re}\left(n_{\infty} i|\xi|\langle\hat{v} \mid \hat{h} \times \omega\rangle\right),
$$

where $\hat{H}_{2}=\operatorname{Re}\langle\hat{E} \mid \hat{h} \times i \omega\rangle$. Thus we arrive at the inequality

$$
\left(|\xi| \hat{H}_{2}\right)_{t}+c_{3}|\xi|^{2}|\hat{h}|^{2} \leq C_{3}|\xi|^{2}|\hat{E}|^{2}+C_{3}|\hat{v}|^{2},
$$

where we used $|\hat{h} \times \omega|=|\hat{h}|$ which is due to (5.15), and $c_{3}$ and $C_{3}$ are positive constants.

Finally, combining (5.16), (5.19) and (5.20), we derive the desired estimate. First we multiply (5.19) and (5.20) by $1+|\xi|^{2}$ and $\beta>0$, respectively, and add these two inequalities. This gives

$$
\begin{gathered}
\left\{\left(1+|\xi|^{2}\right) \hat{H}_{1}+\beta \hat{H}_{2}\right\}_{t}+c_{2}\left(1+|\xi|^{2}\right)^{2}|\hat{\rho}|^{2}+\left\{c_{2}\left(1+|\xi|^{2}\right)-\beta C_{3}|\xi|^{2}\right\}|\hat{E}|^{2} \\
+\left(\beta c_{3}-\varepsilon\right)|\xi|^{2}|\hat{h}|^{2} \leq\left\{C_{\varepsilon}\left(1+|\xi|^{2}\right)^{2}+\beta C_{3}\right\}|\hat{v}|^{2}
\end{gathered}
$$

Next we multiply (5.16) and $(5.21)$ by $\left(1+|\xi|^{2}\right)^{2}$ and $\alpha>0$, respectively, and sum up these two inequalities. Then we arrive at

$$
\begin{aligned}
& \left(1+|\xi|^{2}\right)^{2} \hat{H}_{t}+\left\{c_{0}\left(1+|\xi|^{2}\right)^{2}-\alpha\left\{C_{\varepsilon}\left(1+|\xi|^{2}\right)^{2}+\beta C_{3}\right\}\right\}|\hat{v}|^{2} \\
& +\alpha c_{2}\left(1+|\xi|^{2}\right)^{2}|\hat{\rho}|^{2}+\alpha\left\{c_{2}\left(1+|\xi|^{2}\right)-\beta C_{3}|\xi|^{2}\right\}|\hat{E}|^{2}+\alpha\left(\beta c_{3}-\varepsilon\right)|\xi|^{2}|\hat{h}|^{2} \leq 0
\end{aligned}
$$

where we put

$$
\hat{H}=\hat{H}_{0}+\frac{\alpha}{1+|\xi|^{2}}\left(\hat{H}_{1}+\frac{\beta|\xi|}{1+|\xi|^{2}} \hat{H}_{2}\right)
$$

Let $\beta=c_{2} / 2 C_{3}$ and choose $\varepsilon>0$ such that $\varepsilon=\beta c_{3} / 2$. For this choice of $(\beta, \varepsilon)$, we take $\alpha>0$ suitably small. Then, dividing $(5.22)$ by $\left(1+|\xi|^{2}\right)^{2}$, we reach the inequality

$$
\hat{H}_{t}+c \hat{D} \leq 0
$$

where

$$
\begin{gathered}
c|\hat{z}|^{2} \leq \hat{H} \leq C|\hat{z}|^{2} \\
\hat{D}=|\hat{\rho}|^{2}+|\hat{v}|^{2}+\frac{1}{1+|\xi|^{2}}|\hat{E}|^{2}+\frac{|\xi|^{2}}{\left(1+|\xi|^{2}\right)^{2}}|\hat{h}|^{2} .
\end{gathered}
$$

This shows that $\hat{H}$ in (5.23) is the desired Lyapunov function for our linearized EulerMaxwell system. We see easily that $\hat{D} \geq c \eta(\xi) \hat{H}$, where $\eta(\xi)=|\xi|^{2} /\left(1+|\xi|^{2}\right)^{2}$. Therefore (5.24) gives $\hat{H}_{t}+c \eta(\xi) \hat{H} \leq 0$. Solving this ordinary differential inequality, we obtain $\hat{H}(t, \xi) \leq e^{-c \eta(\xi) t} \hat{H}(0, \xi)$. This together with (5.25) gives the desired pointwise estimate (5.11). This completes the proof of Theorem 5.1.

Proof of Corollary 5.2. By applying the Plancherel theorem and the pointwise estimate (5.11) in Theorem 5.1, we have

$$
\left\|\partial_{x}^{k} e^{t \Phi} \varphi\right\|_{L^{2}}^{2}=\int_{\mathbb{R}^{3}}|\xi|^{2 k}\left|e^{t \hat{\Phi}(i \xi)} \hat{\varphi}(\xi)\right|^{2} d \xi \leq C \int_{\mathbb{R}^{3}}|\xi|^{2 k} e^{-c \eta(\xi) t}|\hat{\varphi}(\xi)|^{2} d \xi
$$


We estimate the right hand side of (5.26). We divide the integral into two parts $R_{1}$ and $R_{2}$ according to the low frequency region $|\xi| \leq 1$ and the high frequency region $|\xi| \geq 1$, respectively. Since $\eta(\xi) \geq c|\xi|^{2}$ for $|\xi| \leq 1$, we find that

$$
R_{1} \leq C \sup _{|\xi| \leq 1}|\hat{\varphi}(\xi)|^{2} \int_{|\xi| \leq 1}|\xi|^{2 k} e^{-c|\xi|^{2} t} d \xi \leq C(1+t)^{-3 / 2+k}\|\varphi\|_{L^{1}}^{2} .
$$

On the other hand, we have $\eta(\xi) \geq c /|\xi|^{2}$ for $|\xi| \geq 1$. Therefore we can estimate $R_{2}$ as

$$
R_{2} \leq C \sup _{|\xi| \geq 1} \frac{e^{-c t /|\xi|^{2}}}{|\xi|^{2 \ell}} \int_{|\xi| \geq 1}|\xi|^{2(k+\ell)}|\hat{\varphi}(\xi)|^{2} d \xi \leq C(1+t)^{-\ell}\left\|\partial_{x}^{k+\ell} \varphi\right\|_{L^{2}}^{2} .
$$

Thus, substituting these estimates into (5.26), we obtain the desired estimate (5.12). This complete the proof of Corollary 5.2.

6. Decay estimate (nonlinear case). The aim of this last section is to prove Theorem 2.3. We need to estimate the time weighted norm $M(t)$ in (2.14). To this end, we introduce

$$
W(t):=\sup _{0 \leq \tau \leq t}(1+\tau)\left\|\left(w-w_{\infty}\right)(\tau)\right\|_{L^{\infty}} .
$$

This norm can be estimated by $M(t)$ as

$$
W(t) \leq C M(t)
$$

for $s \geq 6$. In fact, applying the Gagliardo-Nirenberg inequality, we have

$$
\begin{aligned}
\left\|w-w_{\infty}\right\|_{L^{\infty}} & \leq C\left\|w-w_{\infty}\right\|_{L^{2}}^{1 / 4}\left\|\partial_{x}^{2} w\right\|_{L^{2}}^{3 / 4} \\
& \leq C\left\|w-w_{\infty}\right\|_{H^{s-3}}^{1 / 4}\left\|\partial_{x} w\right\|_{H^{-5}}^{3 / 4} \leq C M(t)(1+t)^{-9 / 8},
\end{aligned}
$$

which gives (6.1), where we assumed $s \geq 6$. We can estimate $M(t)$ in terms of $N(t)$ and $W(t)$ as follows.

Proposition 6.1. Let $s \geq 3$ and suppose that the initial data satisfy $w_{0}-w_{\infty} \in$ $H^{s} \cap L^{1}$ and (1.6). Let $w(t, x)$ be a solution to the problem (1.1), (1.5) satisfying $w-w_{\infty} \in C\left([0, T] ; H^{s}\right) \cap C^{1}\left([0, T] ; H^{s-1}\right)$ and (3.1) for some $T>0$. Then we have

$$
M(t) \leq C I_{1}+C M(t)^{2}+C W(t) N(t),
$$

where $I_{1}=\left\|w-w_{\infty}\right\|_{H^{s}}+\left\|w-w_{\infty}\right\|_{L^{1}}$.

Proof. By making use of the semigroup $e^{t \Phi}$ and applying the Duhamel principle, we can transform (5.2) to the integral equation

$$
z(t)=e^{t \Phi} z_{0}+\int_{0}^{t} e^{(t-\tau) \Phi}\left(A^{0}\right)^{-1}\left(\partial_{x} q+r\right)(\tau) d \tau,
$$

where we simply wrote $\partial_{x} q=\sum_{j=1}^{3} q_{x_{j}}^{j}$. Let $0 \leq k \leq[(s-1) / 2]-1$ and $0 \leq m \leq$ $s-2 k-3$. We apply $\partial_{x}^{k+m}$ to (6.3) and take the $L^{2}$ norm, obtaining

$$
\begin{aligned}
& \left\|\partial_{x}^{k+m} z(t)\right\|_{L^{2}} \leq\left\|\partial_{x}^{k+m} e^{t \Phi} z_{0}\right\|_{L^{2}} \\
& +\left(\int_{0}^{t / 2}+\int_{t / 2}^{t}\right)\left\|\partial_{x}^{k+m} e^{(t-\tau) \Phi}\left(A^{0}\right)^{-1}\left(\partial_{x} q+r\right)(\tau)\right\|_{L^{2}} d \tau=: T_{0}+T_{1}+T_{2} .
\end{aligned}
$$


For the first term $T_{0}$, we apply (5.12) with $\ell=k+2$ to get

$$
\begin{aligned}
T_{0} & \leq C(1+t)^{-3 / 4-(k+m) / 2}\left\|z_{0}\right\|_{L^{1}}+C(1+t)^{-(k+2) / 2}\left\|\partial_{x}^{2 k+2+m} z_{0}\right\|_{L^{2}} \\
& \leq C I_{1}(1+t)^{-3 / 4-k / 2}
\end{aligned}
$$

where we used $\left\|\partial_{x}^{2 k+2+m} z_{0}\right\|_{L^{2}} \leq C\left\|w_{0}-w_{\infty}\right\|_{H^{s-1}} \leq C I_{0}$. Next we estimate the term $T_{1}$. We apply (5.12) with $\ell=k+2, \varphi=\left(A^{0}\right)^{-1} q$ and $\varphi=\left(A^{0}\right)^{-1} r$. This gives

$$
\begin{aligned}
T_{1} & \leq C \int_{0}^{t / 2}(1+t-\tau)^{-5 / 4-(k+m) / 2}\|q(\tau)\|_{L^{1}}+(1+t-\tau)^{-(k+2) / 2}\left\|\partial_{x}^{2 k+3+m} q(\tau)\right\|_{L^{2}} d \tau \\
& +C \int_{0}^{t / 2}(1+t-\tau)^{-3 / 4-(k+m) / 2}\|r(\tau)\|_{L^{1}}+(1+t-\tau)^{-(k+2) / 2}\left\|\partial_{x}^{2 k+2+m} r(\tau)\right\|_{L^{2}} d \tau \\
& \leq C \int_{0}^{t / 2}(1+t-\tau)^{-3 / 4-k / 2}\left\|\left(w-w_{\infty}\right)(\tau)\right\|_{L^{2}}^{2} d \tau \\
& +C \int_{0}^{t / 2}(1+t-\tau)^{-1-k / 2}\left\|\left(w-w_{\infty}\right)(\tau)\right\|_{L^{\infty}}\left\|\partial_{x}^{2 k+2+m} w(\tau)\right\|_{H^{1}} d \tau=: T_{11}+T_{12}
\end{aligned}
$$

where we used the fact that $\left\|\partial_{x}^{j}(p, r)\right\|_{L^{2}} \leq C\left\|w-w_{\infty}\right\|_{L^{\infty}}\left\|\partial_{x}^{j}\left(w-w_{\infty}\right)\right\|_{L^{2}}$ for $0 \leq$ $j \leq s$. Moreover, we have

$$
\begin{aligned}
T_{11} & \leq C M(t)^{2} \int_{0}^{t / 2}(1+t-\tau)^{-3 / 4-k / 2}(1+\tau)^{-3 / 2} d \tau \leq C M(t)^{2}(1+t)^{-3 / 4-k / 2} \\
T_{12} & \leq C W(t) N(t) \int_{0}^{t / 2}(1+t-\tau)^{-1-k / 2}(1+\tau)^{-1} d \tau \\
& \leq C W(t) N(t)(1+t)^{-1-k / 2} \log (1+t)
\end{aligned}
$$

where we used $\left\|\partial_{x}^{2 k+2+m} w\right\|_{H^{1}} \leq\left\|w-w_{\infty}\right\|_{H^{s}} \leq N(t)$. Similarly, we estimate $T_{2}$ by applying (5.12) with $\ell=1, \varphi=\left(A^{0}\right)^{-1} \partial_{x}^{k} q$ and $\varphi=\left(A^{0}\right)^{-1} \partial_{x}^{k} r$. We have

$$
\begin{aligned}
T_{2} & \leq C \int_{t / 2}^{t}(1+t-\tau)^{-5 / 4-m / 2}\left\|\partial_{x}^{k} q(\tau)\right\|_{L^{1}}+(1+t-\tau)^{-1 / 2}\left\|\partial_{x}^{k+2+m} q(\tau)\right\|_{L^{2}} d \tau \\
& +C \int_{t / 2}^{t}(1+t-\tau)^{-3 / 4-m / 2}\left\|\partial_{x}^{k} r(\tau)\right\|_{L^{1}}+(1+t-\tau)^{-1 / 2}\left\|\partial_{x}^{k+1+m} r(\tau)\right\|_{L^{2}} d \tau \\
& \leq C \int_{t / 2}^{t}(1+t-\tau)^{-3 / 4}\left\|\left(w-w_{\infty}\right)(\tau)\right\|_{L^{2}}\left\|\partial_{x}^{k}\left(w-w_{\infty}\right)(\tau)\right\|_{L^{2}} d \tau \\
& +C \int_{t / 2}^{t}(1+t-\tau)^{-1 / 2}\left\|\left(w-w_{\infty}\right)(\tau)\right\|_{L^{\infty}}\left\|\partial_{x}^{k+1+m} w(\tau)\right\|_{H^{1}} d \tau=: T_{21}+T_{22} .
\end{aligned}
$$

Here we see that

$$
\begin{aligned}
T_{21} & \leq C M(t)^{2} \int_{t / 2}^{t}(1+t-\tau)^{-3 / 4}(1+\tau)^{-3 / 2-k / 2} d \tau \leq C M(t)^{2}(1+t)^{-5 / 4-k / 2} \\
T_{22} & \leq C W(t) N(t) \int_{t / 2}^{t}(1+t-\tau)^{-1 / 2}(1+\tau)^{-3 / 2-k / 2} d \tau \\
& \leq C W(t) N(t)(1+t)^{-1-k / 2}
\end{aligned}
$$

where we used the fact that $\left\|\partial_{x}^{k+1+m} w\right\|_{H^{1}} \leq\left\|\partial_{x}^{k+1} w\right\|_{H^{s-2(k+1)}} \leq N(t)(1+\tau)^{-(k+1)}$. 
Finally, we substitute all these estimates into (6.4) and use $\left\|\partial_{x}^{k+m}\left(w-w_{\infty}\right)\right\|_{L^{2}} \leq$ $C\left\|\partial_{x}^{k+m} z\right\|_{L^{2}}$. Then, adding for $m$ with $0 \leq m \leq s-2 k-3$, we arrive at

$$
(1+t)^{3 / 4+k / 2}\left\|\partial_{x}^{k}\left(w-w_{\infty}\right)(t)\right\|_{H^{s-2 k-3}} \leq C I_{0}+C M(t)^{2}+C W(t) N(t)
$$

for $0 \leq k \leq[(s-1) / 2]-1$, from which follows the desired estimate (6.2). This completes the proof of Proposition 6.1.

Finally, to prove the sharper decay estimate (2.17), we introduce the following support norm:

$$
M_{\perp}(t):=\sum_{k=0}^{[(s-1) / 2]-2} \sup _{0 \leq \tau \leq 0}(1+\tau)^{5 / 4+k / 2}\left\|\partial_{x}^{k}\left(n-n_{\infty}, u, E\right)(\tau)\right\|_{H^{s-2 k-5}} .
$$

Proposition 6.2. Let $s \geq 5$. Under the same conditions in Proposition 4.2, we have

$$
M_{\perp}(t) \leq C I_{0}+C M(t)+C W(t) M(t) .
$$

Proof. Similarly as in the derivation of (4.17), we obtain

$$
\begin{gathered}
\left\|\partial_{x}^{k}(u, E)(t)\right\|_{H^{s-2 k-5}} \leq C I_{0} e^{-c t}+C \int_{0}^{t} e^{-c(t-\tau)}\left\|\partial_{x}^{k+1} w(\tau)\right\|_{H^{s-2 k-5}} d \tau \\
+C \int_{0}^{t} e^{-c(t-\tau)}\left\|\left(w-w_{\infty}\right)(\tau)\right\|_{L^{\infty}}\left\|\partial_{x}^{k}\left(w-w_{\infty}\right)(\tau)\right\|_{H^{s-2 k-5}} d \tau
\end{gathered}
$$

for $0 \leq k \leq[(s-1) / 2]-2$. We denote the integrals on the right hand side of (6.6) by $K_{1}$ and $K_{2}$, respectively. Since $\left\|\partial_{x}^{k+1} w(\tau)\right\|_{H^{s-2 k-5}} \leq M(t)(1+\tau)^{-3 / 4-(k+1) / 2}$, we can estimate the term $K_{1}$ as

$$
K_{1} \leq C M(t) \int_{0}^{t} e^{-c(t-\tau)}(1+\tau)^{-5 / 4-k / 2} d \tau \leq C M(t)(1+t)^{-5 / 4-k / 2} .
$$

Also, the term $K_{2}$ is estimated as

$$
K_{2} \leq C W(t) M(t) \int_{0}^{t} e^{-c(t-\tau)}(1+\tau)^{-7 / 4-k / 2} d \tau \leq C W(t) M(t)(1+t)^{-7 / 4-k / 2} .
$$

Thus we obtain

$$
(1+t)^{5 / 4+k / 2}\left\|\partial_{x}^{k}(u, E)(t)\right\|_{H^{s-2 k-5}} \leq C I_{0}+C M(t)+C W(t) M(t)
$$

for $0 \leq k \leq[(s-1) / 2]-2$. On the other hand, by using (1.2), we have

$$
\left\|\partial_{x}^{k}\left(n-n_{\infty}\right)(t)\right\|_{H^{s-2 k-5}} \leq\left\|\partial_{x}^{k+1} E(t)\right\|_{H^{s-2 k-5}} \leq M(t)(1+t)^{-5 / 4-k / 2}
$$

for $0 \leq k \leq[(s-1) / 2]-2$. Thus, combining (6.7) and (6.8), we get the desired estimate (6.5). This completes the proof of Proposition 6.2.

Proof of Theorem 2.3. Let $s \geq 6$. Then we have (6.1). Also we already proved $N(t) \leq C I_{0}$ in (2.10). Substituting these estimates into (6.2), we get

$$
M(t) \leq C I_{1}+C M(t)^{2}+C I_{0} M(t) .
$$


This inequality is solved as $M(t) \leq C I_{1}$, provided that $I_{1}$ is suitably small, say, $I_{1} \leq$ $\varepsilon_{2}$. Thus we have proved (2.15). The decay estimate (2.16) is a direct consequence of (2.15). Moreover, using (6.1) and (2.15) in (6.5), we find that $M_{\perp}(t) \leq C I_{1}$, which gives the decay estimate (2.17). Finally, using (1.2) and (2.17), we obtain

$$
\left\|\partial_{x}^{k}\left(n-n_{\infty}\right)(t)\right\|_{H^{s-2 k-7}} \leq\left\|\partial_{x}^{k+1} E(t)\right\|_{H^{s-2 k-7}} \leq C I_{1}(1+t)^{-7 / 4-k / 2}
$$

for $s \geq 7$ and $0 \leq k \leq[(s-1) / 2]-3$. Thus the proof of Theorem 2.3 is complete.

Acknowledgments. The first author is partially supported by Grant-in-Aid for Young Scientists (B) No. 21740111 from Japan Society for the Promotion of Science. The second author's research is partially supported by Grant-in-Aid for Scientific Research (A) 22244009.

\section{REFERENCES}

[1] S. Bianchini, B. Hanouzet and R. NAtalini, Asymptotic behavior of smooth solutions for partially dissipative hyperbolic systems with a convex entropy, Comm. Pure Appl. Math. 60 (2007), pp. 1559-1622.

[2] F. Chen, Introduction to Plasma Physics and Controlled Fusion, Vol. 1, Plenum Press, New York, 1984.

[3] G. Q. Chen, J. W. Jerome and D. H. Wang, Compressible Euler-Maxwell equations, Transport Theory and Statistical Physics, 29 (2000), pp. 311-331.

[4] C. R. DA Luz AND R. C. CHARÃo, Asymptotic properties for a semilinear plate equation in unbounded domains, J. Hyperbolic Differential Equations, 6 (2009), pp. 269-294.

[5] R. DuAn, Global smooth flows for the compressible Euler-Maxwell system: I. Relaxation case, J. Hyperbolic Differential Equations, 8 (2011), pp. 375-413.

[6] B. Hanouzet AND R. NATAlini, Global existence of smooth solutions for partially dissipative hyperbolic systems with a convex entropy, Arch. Rational Mech. Anal., 169 (2003), pp. 89117.

[7] T. Hosono And S. Kawashima, Decay property of regularity-loss type and application to some nonlinear hyperbolic-elliptic system, Math. Models Meth. Appl. Sci., 16 (2006), pp. 18391859.

[8] K. Ide, K. Haramoto and S. Kawashima, Decay property of regularity-loss type for dissipative Timoshenko system, Math. Models Meth. Appl. Sci., 18 (2008), pp. 647-667.

[9] K. IdE AND S. KaWAShima, Decay property of regularity-loss type and nonlinear effects for dissipative Timoshenko system, Math. Models Meth. Appl. Sci., 18 (2008), pp. 1001-1025.

[10] S. KaWASHIma, Systems of a hyperbolic-parabolic composite type, with applications to the equations of magnetohydrodynamics, Doctoral Thesis, Kyoto University, 1984. http://repository.kulib.kyoto-u.ac.jp/dspace/handle/2433/97887

[11] S. Kawashima And W.-A. Yong, Dissipative structure and entropy for hyperbolic systems of balance laws, Arch. Rational Mech. Anal., 174 (2004), pp. 345-364.

[12] S. Kawashima And W.-A. Yong, Decay estimates for hyperbolic balance laws, ZAA (J. Anal. Appl.), 28 (2009), pp. 1-33.

[13] T. Kubo AND S. KaWAshima, Decay property of regularity-loss type and nonlinear effects for some hyperbolic-elliptic system, Kyushu J. Math., 63 (2009), pp. 1-21.

[14] Y. LIU AND S. KAWASHIMA, Global existence and asymptotic behavior of solutions for quasilinear dissipative plate equation, Discrete Continuous Dynamical Systems, A, 29 (2011), pp. 1113-1139.

[15] Y. LiU AND S. KaWAshima, Global existence and decay of solutions for a quasi-linear dissipative plate equation, J. Hyperbolic Differential Equations, 8 (2011), pp. 591-614.

[16] J. E. MuÑoz Rivera And R. RACKe, Global stability for damped Timoshenko systems, Discrete and Continuous Dynamical Systems, 9 (2003), pp. 1625-1639.

[17] Y. J. PENG AND S. WANG, Rigorous derivation of incompressible e-MHD equations from compressible Euler-Maxwell equations, SIAM J. Math. Anal., 40 (2008), pp. 540-565.

[18] Y. J. PEng AND S. WANG, Asymptotic expansions in two-fluid compressible Euler-Maxwell equations with small parameters, Discrete and Continuous Dynamical Systems, 23 (2009), pp. $415-433$.

[19] Y. J. Peng, S. Wang And Q. L. Gu, Relaxation limit and global existence of smooth solutions of compressible Euler-Maxwell equations, preprint. 
[20] Y. Shizuta And S. Kawashima, Systems of equations of hyperbolic-parabolic type with applications to the discrete Boltzmann equation, Hokkaido Math. J., 14 (1985), pp. 249-275.

[21] Y. Sugitani And S. Kawashima, Decay estimates of solutions to a semi-linear dissipative plate equation, J. Hyperbolic Differential Equations, 7 (2010), pp. 471-501.

[22] T. Umeda, S. Kawashima And Y. Shizuta, On the decay of solutions to the linearized equations of electro-magneto-fluid dynamics, Japan J. Appl. Math., 1 (1984), pp. 435-457.

[23] Y. Ueda, S. Wang and S. KaWashima, Dissipative structure of the regularity-loss type and time asymptotic decay of solutions for the Euler-Maxwell system, to appear in SIAM J. Math. Anal.

[24] W.-A. Yong, Entropy and global existence for hyperbolic balance laws, Arch. Rational Mech. Anal., 172 (2004), pp. 247-266. 
